\title{
A wave propagation method for three-dimensional hyperbolic conservation laws
}

\author{
Jan Olav Langseth * Randall J. LeVeque ${ }^{\dagger}$
}

May 12, 1999

\begin{abstract}
A class of wave propagation algorithms for three-dimensional conservation laws and other hyperbolic systems is developed. These unsplit finite volume methods are based on solving one-dimensional Riemann problems at the cell interfaces and applying flux-limiter functions to suppress oscillations arising from second derivative terms. Waves emanating from the Riemann problem are further split by solving Riemann problems in the transverse directions to model cross-derivative terms. With proper upwinding, a method that is stable for Courant numbers up to one can be developed. Stability theory for three-dimensional algorithms is found to be more subtle than in two dimensions and is studied in detail. In particular we find that some methods which are unconditionally unstable when no limiter is applied are (apparently) stabilized by the limiter function and produce good looking results. Several computations using the Euler equations are presented, including blast wave and complex shock/vorticity problems. These algorithms are implemented in the CLAWPACK software which is freely available.
\end{abstract}

Keywords: Finite-volume methods, high resolution, wave propagation, three dimensions, Euler equations, software.

AMS Classification: 65M06, 76R99

\section{Introduction}

This paper describes an unsplit method for solving three-dimensional conservation laws, i.e. equations of the form

$$
q_{t}+f(q)_{x}+g(q)_{y}+h(q)_{z}=0
$$

where $q \in \mathbb{R}^{m}$ is the conserved quantity. It is well known that these equations may develop shock waves or contain other discontinuities. The existence of regions where the solution changes abruptly places special requirements on the numerical methods to be used. Traditionally, simple numerical schemes suffer from dissipative and dispersive effects, resulting in

\footnotetext{
${ }^{*}$ Norwegian Defence Research Establishment, P.O.Box 25, N-2027 Kjeller, Norway. This work has been supported by The Norwegian Research Council (NFR) through the program no. 101039/420.

${ }^{\dagger}$ Departments of Mathematics and Applied Mathematics, University of Washington, Seattle, WA 981952420. This work has been supported by DOE grant DE-FG03-96ER25292 and NSF grants DMS-9505021 and DMS-9626645.
} 
inappropriate representation of these discontinuities. This is reflected in the solution as spurious oscillations or excessive smearing in the vicinity of the discontinuity. Since the occurrence of discontinuous waves is a significant feature of hyperbolic problems, much effort has been made in order to construct robust methods producing sharp and monotone representations.

One special initial value problem is of major importance, both in its own and in the development of efficient numerical methods. This is the Riemann problem which consists of the equation $q_{t}+f(q)_{x}=0$ together with the initial condition

$$
q(x, 0)= \begin{cases}q_{l} & x<0 \\ q_{r} & x \geq 0\end{cases}
$$

where $q_{l}$ and $q_{r}$ are constants. With certain assumptions on the flux function $f$, it is always possible, in principle, to solve the Riemann problem if the states $q_{l}$ and $q_{r}$ are sufficiently "close", see [20], [38]. The solution consists of waves travelling with finite velocities. These waves may either be discontinuous waves like shock waves or smooth rarefaction waves. The similarity solution of this initial-value problem depends on the ratio $x / t$. Due to this simplification, it is possible to solve any scalar problem. Also for many important nonlinear systems, the Riemann problem can be solved, e.g., the Euler equations of gas dynamics [15], [38]. The procedure for constructing the solution of a Riemann problem will be called a Riemann solver.

The history of the development of numerical methods for hyperbolic conservation laws is long and rich. The wave propagation method described in this paper falls into the tradition of methods based on solving Riemann problems which originated with the work of Godunov[14]. For a general overview of such methods and many references, see for example [13], [17], [23], [43].

A common approach when solving multi-dimensional hyperbolic problems is to apply dimensional splitting, see [9],[40]. The idea is to iterate on one-dimensional problems. The popularity of these algorithms is due to their simplicity and the fact that they produce surprisingly good results. Any one-dimensional scheme is easily extended to the multi-dimensional case using this approach. However, it is well known that dimensional splitting has several disadvantages. Since the strategy only involves flow in the coordinate directions, the solution is affected by the grid orientation. Discontinuities travelling obliquely to the grid experience more smearing than those travelling in the coordinate directions. The implementation of boundary conditions may also be complicated using this strategy.

In unsplit methods, information is propagated in a multi-dimensional way. The unsplit scheme to be presented was first described in an unfinished form in [18], and later in a state close to the present, in the thesis of the first author [19]. One-dimensional Riemann problems are solved at the interfaces. Limiter functions are applied to suppress spurious oscillations arising from second derivative terms. The left-going and right-going waves are split into parts propagating in the transverse direction by solving Riemann problems in coordinate directions tangential to the interfaces. This models cross-derivative terms necessary for obtaining both a stable and formally second order scheme. The scheme extends the approach used for two spacedimensions [24], [26], and the advection scheme for three-dimensional problems considered in [25]. The 3D wave propagation scheme is implemented in Fortran, and is included in the software package CLAWPACK (Conservation LAW PACKage) [27]. This package includes routines for solving a wide range of hyperbolic problems, and contains a selection of different Riemann solvers, boundary conditions etc. CLAWPACK is freely available on the Web [27]. 
This method is similar in spirit to other multi-dimensional methods using one-dimensional Riemann problems at the cell interfaces, see [2], [5], [7], [22], [29], [32], [35], [37], [44]. However, an algorithmic difference is that the Riemann problem is based on the solution values at the beginning of the time step, and that cross-derivative terms are implemented due to the transverse propagation of the emanating waves. In other methods, such as that of Colella [7], the states involved in the Riemann problem are based on an interpolation in which the transverse terms are included before solving the Riemann problem.

In the wave propagation algorithm, it is not necessary to evaluate the flux functions explicitly. As a consequence, the algorithm may as well be applied on systems in non-conservative form. In CLAWPACK, the scheme is implemented so that it is applicable on quasilinear problems having the form

$$
\kappa(x, y, z) q_{t}+A(q, x, y, z, t) q_{x}+B(q, x, y, z, t) q_{y}+C(q, x, y, z, t) q_{z}=\Psi(q, \kappa, x, y, z, t) .
$$

Recent work on acoustic and elastic waves in heterogeneous media [11], [12] shows that these methods can be extremely useful even for linear problems since the solution of the Riemann problem accurately models the transmission and reflection of waves at a material interface. The three-dimensional algorithm developed here should be applicable to problems of this type as well. In this paper, we focus on the conservation law (2), and refer to [26] for a discussion of how to apply CLAWPACK routines to this more general class of problems. In Section 2, the wave propagation scheme is derived. In the process of deriving this scheme, the one-dimensional and two-dimensional versions are briefly reviewed. In Section 3, numerical results are given for three test problems involving the Euler equations: a radially symmetric smooth solution where second order accuracy can be verified, an initially spherical shock wave expanding in a slab between two parallel walls, and finally a fully 3D problem, in which shock waves are used to produce vorticity.

Stability is the topic of Section 4. A von Neumann approach is used to shed some light on the question of stability for the wave propagation scheme and how the use of limiters affects this property. With proper upwinding, a method that is stable for Courant numbers up to one can be developed. However, stability theory for three-dimensional algorithms is found to be more subtle than in two dimensions and we study this in detail. In particular we find that some methods which are unconditionally unstable when no limiter is applied are (apparently) stabilized by the limiter function and produce good looking results.

\section{Wave propagation algorithms}

In this section we will derive the three-dimensional wave propagation scheme. This will generalize the schemes for two-dimensional systems described in [26] and scalar three-dimensional problems from [25]. We give a brief review of the one- and two-dimensional wave-propagation methods, but in order to avoid substantial repetition we assume that the reader is familiar with the notation and ideas of [25] and [26].

We assume that the three-dimensional system of conservation laws (1) is hyperbolic, i.e. that the matrix $\alpha f^{\prime}(q)+\beta g^{\prime}(q)+\gamma h^{\prime}(q)$ has real eigenvalues and a complete set of eigenvectors

for any real $\alpha, \beta$, and $\gamma$ with $|\alpha|+|\beta|+|\gamma|>0$. Define a regular grid with constant spacing $\Delta x, \Delta y$, and $\Delta z$. Let $C_{i j k}$ denote the cell $\left[x_{i}, x_{i+1}\right] \times\left[y_{j}, y_{j+1}\right] \times\left[z_{k}, z_{k+1}\right]$, where $x_{i}=i \Delta x$, etc. Likewise let $\Delta t$ denote the time step, and $t_{n}=n \Delta t$ the time levels. 
In order to derive the numerical scheme we primarily consider two linear problems, namely the scalar equation

$$
q_{t}+u q_{x}+v q_{y}+w q_{z}=0
$$

with positive velocities, and the linear system

$$
q_{t}+A q_{x}+B q_{y}+C q_{z}=0 .
$$

The essential ideas and difficulties in three-dimensional wave-propagation appear already with these linear problems. The extension to nonlinear systems is then immediate following the approach used already in two space dimensions in [26].

The wave propagation schemes will be written in the same form as in that paper,

$$
\bar{Q}_{i j k}=Q_{i j k}+\Delta_{i j k}^{u p}-\frac{\Delta t}{\Delta x}\left(\tilde{F}_{i+1, j k}-\tilde{F}_{i j k}\right)-\frac{\Delta t}{\Delta y}\left(\tilde{G}_{i, j+1, k}-\tilde{G}_{i j k}\right)-\frac{\Delta t}{\Delta z}\left(\tilde{H}_{i j, k+1}-\tilde{H}_{i j k}\right),
$$

where $\bar{Q}$ represents the numerical solution at the time step $t_{n+1}$. The term $\Delta_{i j k}^{u p}$ includes the donor-cell part, i.e. a (one-dimensional) first order upwind scheme applied coordinate-wise. The $\tilde{F}, \tilde{G}$, and $\tilde{H}$ terms take care of the approximations of the higher order derivatives, including cross-derivative terms. The flux functions will not be needed explicitly, and as a consequence, the scheme can easily be applied to a wider range of hyperbolic problems.

\subsection{Schemes for linear 1D problems}

Essential features of the wave propagation schemes are the so-called increment and correction wave. The former refers to the constant state waves given from the solution of the (approximate) Riemann problem, while the correction waves are piecewise linear waves used for obtaining second order accuracy. These features are best illustrated by using the simple scalar advection equation

$$
q_{t}+u q_{x}=0, \quad u>0 .
$$

The conservative scheme for one-dimensional problems reads

$$
\bar{Q}_{i}=Q_{i}-\frac{\Delta t}{\Delta x}\left(F_{i+1}-F_{i}\right),
$$

where the numerical flux $F_{i}$ approximates the exact flux, i.e.

$$
F_{i} \approx \frac{1}{\Delta t} \int_{t_{n}}^{t_{n+1}} f\left(q\left(x_{i}, \cdot\right)\right) d t
$$

As in standard second order Godunov methods $([23],[46])$, assume that the solution at time $t_{n}$ is piecewise linear. The solution at time $t_{n+1}$ is obtained by simply shifting the profile a distance $u \Delta t$. The situation close to the interface $x_{i}$ then looks as depicted in Figure 1. Since the problem is linear, the wave entering cell $C_{i}$ may be split into a piecewise constant and a piecewise linear wave. These waves are named increment and correction waves respectively. It is easily seen that the numerical flux in this case reads

$$
F_{i}=u Q_{i-1}+\frac{1}{2} u\left(1-u \frac{\Delta t}{\Delta x}\right) \Delta x \sigma_{i-1}
$$



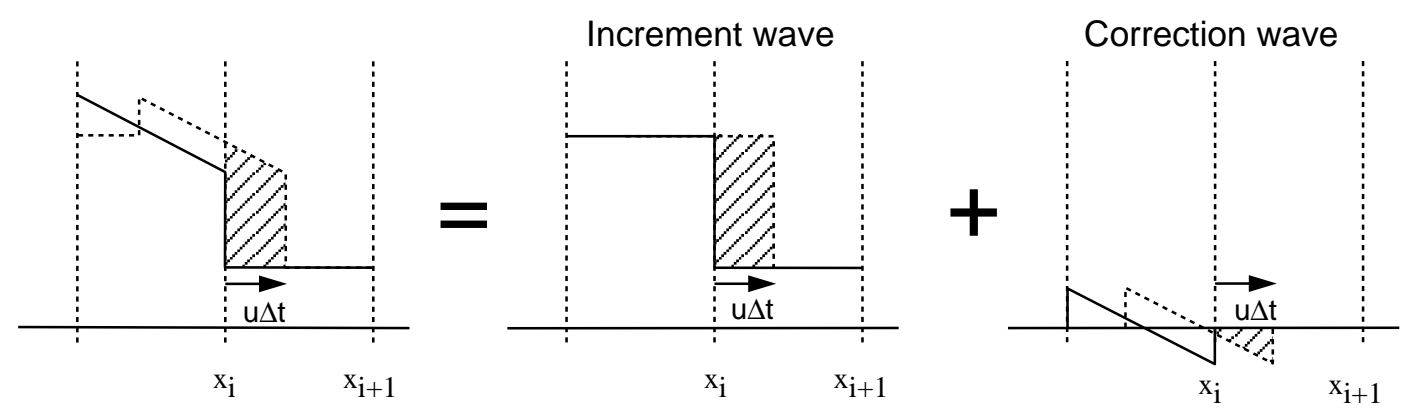

Figure 1: The piecewise linear function can be split into a constant state wave (the increment wave) and a piecewise linear wave with integral zero (the correction wave).

where $\sigma_{i-1}$ denotes the slope of the solution in cell $C_{i-1}$. The first term at the right hand side of (9) corresponds to the flux contribution from the increment wave, while the last term is the contribution from the correction wave. Note that the increment wave alone yields the first order upwind scheme and that the correction wave may correct this into a second order scheme if the slope is chosen correctly.

Next, consider the linear system $f(q)=A q$, where $A$ is a constant $m \times m$ matrix with eigenvalues $\lambda^{p}$ and eigenvectors $r^{p}$. Since $A$ has a complete set of eigenvectors, the matrix is diagonalizable, i.e.

$$
A=R \Lambda R^{-1}
$$

where $R=\left[r^{1}\left|r^{2}\right| \ldots \mid r^{m}\right]$ is the eigenvector matrix and $\Lambda=\operatorname{diag}\left(\lambda^{1}, \lambda^{2}, \ldots, \lambda^{m}\right)$. For $a \in \mathbb{R}$ let

$$
a^{+}=\max (0, a), \quad a^{-}=\min (0, a) .
$$

Define the positive and negative parts of $A$ as

$$
A^{+}=R \Lambda^{+} R^{-1}, \quad A^{-}=R \Lambda^{-} R^{-1},
$$

where $\Lambda^{ \pm}=\operatorname{diag}\left(\lambda^{1 \pm}, \lambda^{2 \pm}, \ldots, \lambda^{m \pm}\right)$.

The solution of the Riemann problem defined by $Q_{i-1}$ and $Q_{i}$ consists of $m$ discontinuities moving with velocities $\lambda^{p}$. Each jump is a scalar multiple of the eigenvector, i.e. $\mathcal{W}_{i}^{p}=\alpha_{i}^{p} r^{p}$. Hence the jump $\Delta Q_{i}=Q_{i}-Q_{i-1}$ may be written as

$$
\Delta Q_{i}=\sum_{p} \mathcal{W}_{i}^{p}
$$

The first order Godunov flux is defined as

$$
F_{i}^{G}=f\left(Q_{i}^{0}\right),
$$

where $Q_{i}^{0}$ denotes the solution at $x / t=0$ of the Riemann problem with left state $Q_{i-1}$ and right state $Q_{i}$. For the linear system this intermediate state equals

$$
Q_{i}^{0}=Q_{i-1}+\sum_{\lambda^{p}<0} \mathcal{W}_{i}^{p}=Q_{i}-\sum_{\lambda^{p}>0} \mathcal{W}_{i}^{p}
$$

Hence, the Godunov flux reads

$$
F_{i}^{G}=A Q_{i-1}+A^{-} \Delta Q_{i}=A Q_{i}-A^{+} \Delta Q_{i} .
$$


For the numerical scheme (8) we get

$$
\bar{Q}_{i}=Q_{i}-\frac{\Delta t}{\Delta x}\left(A^{+} \Delta Q_{i}+A^{-} \Delta Q_{i+1}\right) .
$$

The expression $A^{+} \Delta Q_{i}=\sum_{p}\left(\lambda^{p}\right)^{+} \mathcal{W}_{i}^{p}$ gives the flux contribution from the waves entering cell $C_{i}$ from the Riemann problem at the left interface, while $A^{-} \Delta Q_{i+1}$ gives the flux from the waves entering the same cell from the Riemann problem at the right interface. These piecewise constant waves, emanating from the Riemann problems, will be named increment waves, as a generalization of similar waves in the scalar case.

\subsection{Second order corrections}

The scheme considered above is first order accurate only. In order to increase the order, (8) is rewritten as

$$
\bar{Q}_{i}=Q_{i}+\Delta_{i}^{u p}-\frac{\Delta t}{\Delta x}\left(\widetilde{F}_{i+1}-\widetilde{F}_{i}\right)
$$

where $\Delta_{i}^{u p}$ equals the upwind flux (or Godunov flux) obtained from (11), i.e.

$$
\Delta_{i}^{u p}=-\frac{\Delta t}{\Delta x}\left(A^{+} \Delta Q_{i}+A^{-} \Delta Q_{i+1}\right) .
$$

The term $\widetilde{F_{i}}$ is used to update the solution so that second order accuracy is achieved. This is based on the Lax-Wendroff scheme, though in Section 4 we will discuss the possible merits of using other second order schemes as the starting point.

The flux for the Lax-Wendroff scheme may be written as follows:

$$
F_{i}=F_{i}^{G}+\frac{1}{2}|A|\left(1-\frac{\Delta t}{\Delta x}|A|\right) \Delta Q_{i}
$$

where $|A|=A^{+}-A^{-}$. Hence, a natural choice for $\widetilde{F_{i}}$ is

$$
\begin{aligned}
\widetilde{F_{i}} & =\frac{1}{2}|A|\left(1-\frac{\Delta t}{\Delta x}|A|\right) \Delta Q_{i} \\
& =\frac{1}{2} \sum_{p}\left|\lambda^{p}\right|\left(1-\frac{\Delta t}{\Delta x}\left|\lambda^{p}\right|\right) \mathcal{W}_{i}^{p} .
\end{aligned}
$$

The Godunov scheme exhibits strong numerical dissipation, and discontinuities in the solution are smeared causing low accuracy. The Lax-Wendroff scheme, on the other hand, is more accurate in smooth parts of the solution. But near discontinuities, numerical dispersion generates oscillations, also reducing the accuracy.

A successful approach to suppress these oscillations is to apply flux limiting. This is obtained by replacing the wave $\mathcal{W}_{i}^{p}=\alpha_{i}^{p} r^{p}$ by

$$
\widetilde{\mathcal{W}}_{i}^{p}=\Phi\left(\theta_{i}^{p}\right) \mathcal{W}_{i}^{p}
$$

where $\theta_{i}^{p}$ measures the smoothness of the solution. A standard way of doing this is to consider the ratio of wave strengths $\alpha_{i}^{p}$ in the upwind direction, i.e.

$$
\theta_{i}^{p}= \begin{cases}\alpha_{i-1}^{p} / \alpha_{i}^{p}, & \lambda^{p}>0 \\ \alpha_{i+1}^{p} / \alpha_{i}^{p}, & \lambda^{p}<0\end{cases}
$$


The limiters used here are originally constructed in order to ensure both second order accuracy and TVD properties for scalar conservation laws. For a detailed discussion of such methods, see [13], [23], [42], [43]. The TVD concept is not applicable in the system case directly, but applying this strategy on the characteristic equations, as done implicitly here, produces steep and monotone approximations of discontinuities.

Note that $\Phi=1$ yields the Lax-Wendroff scheme, while $\Phi=0$ yields the Godunov scheme. In Section 4.3 we are also going to study the Fromm scheme and the Beam-Warming scheme, both being second order accurate. Here we simply note that $\Phi=\theta$ gives the latter scheme, while $\Phi=(1+\theta) / 2$ gives the former. Some common limiters are:

$$
\begin{aligned}
\text { minmod: } & \Phi(\theta)=\max (0, \min (1, \theta)) \\
\text { superbee: } & \Phi(\theta)=\max (0, \min (1,2 \theta), \min (2, \theta)) \\
\text { monotonized centered }(\mathrm{MC}): & \Phi(\theta)=\max (0, \min ((1+\theta) / 2,2,2 \theta))
\end{aligned}
$$

Minmod is the most diffusive limiter of the above, in the sense that it adds less downwind contribution. This limiter selects the wave with the smallest norm of the two compared, provided the jumps across the waves are in the same direction, i.e. the wave strengths have common sign. If not, the wave is entirely suppressed. On the other hand, the superbee limiter is known to be "overcompressive", i.e. it tends to sharpen profiles into discontinuities. The MC-limiter seems to be a good choice in most situations.

As a generalization of the correction waves in the scalar case, the piecewise linear waves used in the second order update will also be referred to as correction waves.

\subsection{The propagation of the increment wave}

Also in the three-dimensional case, we solve one-dimensional Riemann problems. The structure of multi-dimensional Riemann problems is so complex that it is not beneficial to use them as building blocks in a numerical scheme, cf. [36]. The increment and correction waves in 3D are simple extensions of those obtained in 1D. Hence, waves originating from an interface in $x$ are functions of $x$ and $t$ only. The description below will focus primarily on the solution of the Riemann problem defined at the interface $x_{i}$, and how the emanating waves update the upwind term $\Delta_{i j k}^{u p}, \widetilde{F}_{i j k}$ and nearby $\widetilde{G}$ and $\widetilde{H}$ fluxes. An analogous procedure is followed at each interface in the $y$-direction and in the $z$-direction.

Propagating the waves in different ways transverse to the interface leads to a family of possible methods with different accuracy and stability properties. These will be built up over the next several sections. Stability analysis is presented in Section 4.

\subsubsection{Scalar equation}

For the scalar equation (4), a simple first order scheme can be made by considering first order upwind terms in each coordinate direction. This yields a donor-cell type of scheme in which the upwind part reads

$$
\Delta_{i j k}^{u p}=-\frac{\Delta t}{\Delta x} u Q_{i-1, j k}-\frac{\Delta t}{\Delta y} v Q_{i, j-1, k}-\frac{\Delta t}{\Delta z} w Q_{i j, k-1} .
$$




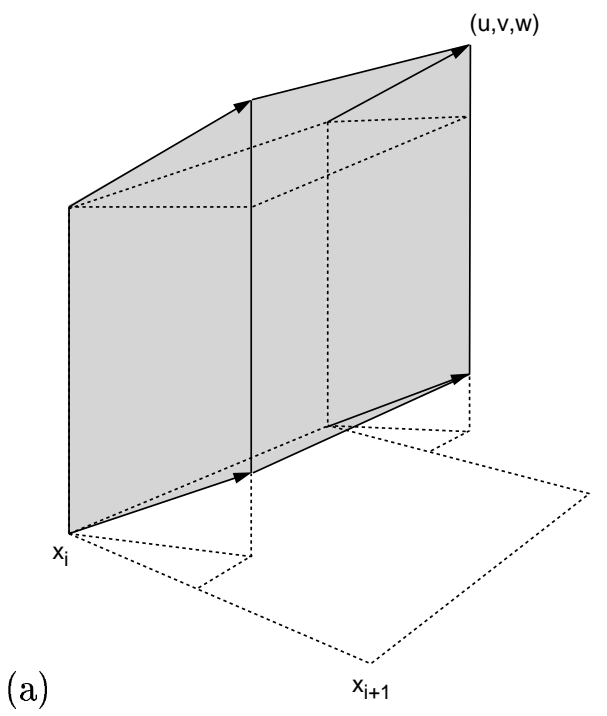

(b)

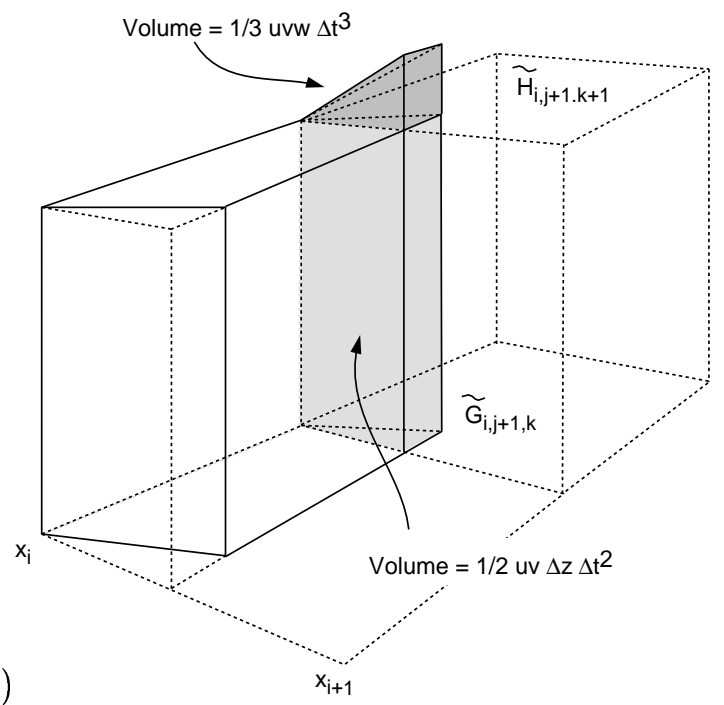

Figure 2: (a) The volume covered by the increment wave, when the velocities are positive. (b) The volume used in updating the cells $C_{i j, k+1}$ and $C_{i, j+1, k+1}$.

The stability condition of this scheme is easily seen to be $u \frac{\Delta t}{\Delta x}+v \frac{\Delta t}{\Delta y}+w \frac{\Delta t}{\Delta z} \leq 1$ (see Section 4). In order to increase both stability and accuracy, the increment wave is advected in the direction of the velocity vector $(u, v, w)$ (Figure 2). This yields the "shift and average" scheme, in which the piecewise constant function defined by the cell values $Q_{i j k}$ is shifted according to the velocity, and then averaged back onto the grid. This method is clearly stable for Courant numbers up to 1 .

To see how the "shift and average" scheme is defined in a flux formulation, consider the updates of the $\widetilde{G}_{i, j+1, k}$ given by the increment wave originating from the interface $x=x_{i}$. (The wave is defined by the jump $-\Delta_{x} Q_{i j k}=Q_{i-1, j, k}-Q_{i j k}$ and the velocity vector.)

$$
\begin{aligned}
\widetilde{G}_{i, j+1, k} & :=\widetilde{G}_{i, j+1, k}-\frac{1}{\Delta t} \frac{1}{\Delta z} \frac{1}{\Delta x} \int_{t_{n}}^{t_{n+1}} \int_{z_{k}+w\left(t-t_{n}\right)}^{z_{k+1}} \int_{x_{i}}^{x_{i}+u\left(t-t_{n}\right)} v \Delta_{x} Q_{i j k} d x d z d t, \\
& =\widetilde{G}_{i, j+1, k}-\frac{1}{2} \frac{\Delta t}{\Delta x} u v \Delta_{x} Q_{i j k}+\frac{1}{3} u v w \frac{\Delta t}{\Delta x} \frac{\Delta t}{\Delta z} \Delta_{x} Q_{i j k} .
\end{aligned}
$$

Another way of deriving this expression is to consider the volume in cell $C_{i, j+1, k}$ covered by the wave, which equals $\frac{1}{2} u v \Delta t^{2} \Delta z-\frac{1}{3} u v w \Delta t^{3}$. Since the wave carries the constant jump $-\Delta_{x} Q_{i j}$, the appropriate flux update equals (17). Likewise, the volume covered by this wave in the other neighboring cells are

$$
\begin{aligned}
C_{i j, k+1}: & \frac{1}{2} u v \Delta t^{2} \Delta z-\frac{1}{3} u v w \Delta t^{3} \\
C_{i, j+1, k+1}: & \frac{1}{3} u v w \Delta t^{3}
\end{aligned}
$$

These volumes are shown in Figure $2 \mathrm{~b}$. The actual change in the solution equals these volumes times the jump $-\Delta_{x} Q_{i j k}$, and are obtained by updating the fluxes. There are several ways of distributing the solution updates between the fluxes in the scalar case. Here, a different approach than in [25] will be used, since this will be in agreement with the appropriate terms in the system case. 
When the velocities are positive, the flux updates are

$$
\begin{aligned}
\widetilde{G}_{i, j+1, k} & :=\widetilde{G}_{i, j+1, k}-\frac{1}{2} u v \frac{\Delta t}{\Delta x} \Delta_{x} Q_{i j k}+\frac{1}{6} u v w \frac{\Delta t}{\Delta x} \frac{\Delta t}{\Delta z} \Delta_{x} Q_{i j k} \\
\widetilde{G}_{i, j+1, k+1} & :=\widetilde{G}_{i, j+1, k+1}-\frac{1}{6} u v w \frac{\Delta t}{\Delta x} \frac{\Delta t}{\Delta z} \Delta_{x} Q_{i j k} \\
\widetilde{H}_{i j, k+1} & :=\widetilde{H}_{i j, k+1}-\frac{1}{2} u v \frac{\Delta t}{\Delta x} \Delta_{x} Q_{i j k}+\frac{1}{6} u v w \frac{\Delta t}{\Delta x} \frac{\Delta t}{\Delta y} \Delta_{x} Q_{i j k} \\
\widetilde{H}_{i, j+1, k+1} & :=\widetilde{H}_{i, j+1, k+1}-\frac{1}{6} u v w \frac{\Delta t}{\Delta x} \frac{\Delta t}{\Delta y} \Delta_{x} Q_{i j k} .
\end{aligned}
$$

Doing the same for increment waves originating from interfaces in $y$ and $z$ gives a scheme identical to the "shift and average" scheme. Hence, we have obtained a first order scheme with the desired stability limit, i.e. stability for Courant numbers less than or equal to one, as described further in Section 4.

Note that the update of $\widetilde{G}_{i, j+1, k}$ consists of two terms. The first term, also found in the two-dimensional wave propagation schemes, cf. [26], corresponds to the part of the wave moving into cells sharing an interface with either of the cells defining the Riemann problem, i.e. $C_{i-1, j k}$ and $C_{i j k}$. This feature will be named transverse propagation. The last update, named double transverse propagation, is a pure three-dimensional contribution, and is caused by the part of the wave moving into cells only sharing an edge with one of these cells.

Also note that the updates of $\widetilde{G}$ and $\widetilde{H}$ above contains a difference in $x$. Hence these updates will account for terms like $\frac{1}{2} u v q_{x y}$ and $\frac{1}{6} u v w q_{x y z}$ in a Taylor expansion. This will be studied to some detail in the next section.

\subsubsection{Linear systems}

Here we consider the propagation of the increment waves for the linear system (5). Since the problem is assumed to be hyperbolic, $A, B$, and $C$ are diagonalizable. We define the positive and negative parts of $B$ similar to what was done for $A$ in (10), i.e.

$$
B^{ \pm}=W M^{ \pm} W^{-1}
$$

where $W$ is the eigenvector matrix, and $M$ the diagonal eigenvalue matrix. The eigenvalues are $\mu^{q}$, and the associated eigenvectors $w^{q}$. Likewise, let $\nu^{l}$ and $s^{l}$ denote the eigenvalues and eigenvectors of $C$. Then we define

$$
C^{ \pm}=S N^{ \pm} S^{-1}
$$

where $S$ is the eigenvector matrix and $N$ the diagonal matrix with the eigenvalues $\nu^{l}$ as entries.

Before proceeding with the three-dimensional propagation of the increment wave, we expand $q(x, y, z, t+\Delta t)$ in a Taylor series including terms up to third order in $\Delta t$. This is useful for understanding how the specific wave propagation affects the solution. Due to the large number of terms, we only consider those differentiated first in $x$.

The flux updates resulting from a single interface in $x$ should lead to approximations of these terms, which are

$$
\begin{aligned}
& \Delta t A q_{x}+\frac{1}{2} \Delta t^{2}\left(A^{2} q_{x x}+B A q_{x y}+C A q_{x z}\right)-\frac{1}{6} \Delta t^{3}\left(A^{3} q_{x x x}+A B A q_{x y x}+\right. \\
& \left.A C A q_{x z x}+B A^{2} q_{x x y}+B^{2} A q_{x y y}+B C A q_{x z y}+C A^{2} q_{x x z}+C B A q_{x y z}+C^{2} A q_{x z z}\right) .
\end{aligned}
$$


The (full) wave propagation scheme to be derived will cover all these terms, except for the $A^{3} q_{x x x}$ term. In addition, some fourth order terms will also be accounted for. This is necessary for stability, as discussed in Section 4.

Unless the matrices have a common set of eigenvectors it is not possible to decompose (5) into a system of $m$ scalar advection equations. Nevertheless, it is useful to talk about wave propagation locally even in the general case.

The first step in the algorithm is to solve the one dimensional Riemann problem normal to the interface, i.e. find (increment) waves $\mathcal{W}_{i j k}^{p}$ so that $Q_{i j k}-Q_{i-1, j k}=\sum_{p} \mathcal{W}_{i j k}^{p}$. This is exactly the same approach as used in the one-dimensional case. For example,

$$
A^{-} \Delta_{x} Q_{i j k}=\sum\left(\lambda^{p}\right)^{-} \mathcal{W}_{i j k}^{p}, \quad A^{+} \Delta_{x} Q_{i j}=\sum\left(\lambda^{p}\right)^{+} \mathcal{W}_{i j}^{p}
$$

and

$$
\Delta_{i j k}^{u p}:=\Delta_{i j k}^{u p}-\frac{\Delta t}{\Delta x}\left(A^{-} \Delta_{x} Q_{i+1, j k}+A^{+} \Delta_{x} Q_{i j}\right) .
$$

Doing the same in the $y$ and $z$ directions yields a scheme in which waves only propagate normal to the cell interfaces, i.e. the donor-cell approach.

Next, every increment wave from the Riemann problem in $x$ is split into waves moving in the $y$-direction, i.e.

$$
\mathcal{W}_{i j k}^{p}=\sum_{q} \beta_{i j k}^{p q} w^{q} .
$$

Each $\mathcal{W}_{i j k}^{p}$ will then update any of the surrounding $\widetilde{G}$ fluxes depending on the sign of $\lambda^{p}$. For example, if $\lambda^{p}$ and $\mu^{q}$ are both positive, then

$$
\widetilde{G}_{i, j+1, k}:=\widetilde{G}_{i, j+1, k}-\frac{1}{2} \frac{\Delta t}{\Delta x} \lambda^{p} \mu^{q} \beta_{i j k}^{p q} w^{q},
$$

similar to the first term in the update in (17). The sum of all contributions to this flux gives

$$
\widetilde{G}_{i, j+1, k}:=\widetilde{G}_{i, j+1, k}-\frac{1}{2} \frac{\Delta t}{\Delta x} \sum_{q} \sum_{p}\left(\lambda^{p}\right)^{+}\left(\mu^{q}\right)^{+} \beta_{i j}^{p q k} w^{q} .
$$

It is easily seen that this is equal to

$$
\widetilde{G}_{i, j+1, k}:=\widetilde{G}_{i, j+1, k}-\frac{1}{2} \frac{\Delta t}{\Delta x} B^{+} A^{+} \Delta_{x} Q_{i j k} .
$$

The proper updates for the rest of the $\widetilde{G}$-fluxes are

$$
\begin{aligned}
\widetilde{G}_{i-1, j, k} & :=\widetilde{G}_{i-1, j, k}-\frac{1}{2} \frac{\Delta t}{\Delta x} B^{-} A^{-} \Delta_{x} Q_{i j k} \\
\widetilde{G}_{i j k} & :=\widetilde{G}_{i j k}-\frac{1}{2} \frac{\Delta t}{\Delta x} B^{-} A^{+} \Delta_{x} Q_{i j k} \\
\widetilde{G}_{i-1, j+1, k} & :=\widetilde{G}_{i-1, j+1, k}-\frac{1}{2} \frac{\Delta t}{\Delta x} B^{+} A^{-} \Delta_{x} Q_{i j k},
\end{aligned}
$$

Exactly the same terms are found in the two-dimensional scheme described in [26].

Note that since

$$
B^{+} A^{+}+B^{-} A^{+}+B^{+} A^{-}+B^{-} A^{-}=B A
$$


the flux updates above will result in an approximation to the $\frac{1}{2} \Delta t^{2} B A q_{x y}$ term in the Taylor expansion.

Next, consider transverse propagation of the increment wave in the $z$-direction. This results in an update of the $\widetilde{H}$ fluxes similar to (24) and (25),

$$
\begin{aligned}
\widetilde{H}_{i-1, j k} & :=\widetilde{H}_{i-1, j k}-\frac{1}{2} \frac{\Delta t}{\Delta x} C^{-} A^{-} \Delta_{x} Q_{i j k} \\
\widetilde{H}_{i j k} & :=\widetilde{H}_{i j k}-\frac{1}{2} \frac{\Delta t}{\Delta x} C^{-} A^{+} \Delta_{x} Q_{i j k} \\
\widetilde{H}_{i j, k+1} & :=\widetilde{H}_{i j, k+1}-\frac{1}{2} \frac{\Delta t}{\Delta x} C^{+} A^{+} \Delta_{x} Q_{i j k} \\
\widetilde{H}_{i-1, j, k+1} & :=\widetilde{H}_{i-1, j, k+1}-\frac{1}{2} \frac{\Delta t}{\Delta x} C^{+} A^{-} \Delta_{x} Q_{i j k},
\end{aligned}
$$

which account for $\frac{1}{2} \Delta t^{2} C A q_{x z}$ in the Taylor series.

In an implementation, updates like (23) would require the solution of $m$ Riemann problems, in addition to the one necessary for obtaining $\mathcal{W}^{p}$. In general, this involves too much work. It is also in general too expensive to derive the $A^{ \pm}$and $B^{ \pm}$matrices explicitly, and then perform matrix-vector multiplications. Instead the left-going flux difference $A^{-} \Delta_{x} Q_{i j}$ and the rightgoing flux difference $A^{+} \Delta_{x} Q_{i j}$ are split into eigenvectors $w^{q}$ of $B$, yielding transverse-moving waves. This transverse flux difference splitting is obtained by solving the following equations for $\delta_{i j k}^{q}$

$$
A^{-} \Delta_{x} Q_{i j k}=\sum_{q}\left(\delta_{i j k}^{q}\right)^{-} w^{q}, \quad A^{+} \Delta_{x} Q_{i j k}=\sum_{q}\left(\delta_{i j k}^{q}\right)^{+} w^{q} .
$$

The computations of the flux updates in (24) and (25) are significantly simplified, for example

$$
B^{+} A^{+} \Delta_{x} Q_{i j k}=\sum_{q}\left(\mu^{q}\right)^{+}\left(\delta_{i j k}^{q}\right)^{+} w^{q},
$$

with similar expressions for the rest of the updates. (The number of Riemann problems per interface is now five, regardless of the size of the system.)

Comparing with (18), the updates caused by the increment waves do not, so far, account for full three-dimensional propagation. To do this, the waves should also move into cells only sharing an edge with the cells defining the Riemann problem, i.e. double transverse propagation. This yields approximations of cross-derivatives like $\frac{1}{6} \Delta t^{3} C B A q_{x y z}$. For this term, the proper updates can be shown to be

$$
\begin{aligned}
\widetilde{H}_{i j, k+1} & :=\widetilde{H}_{i j, k+1}+\frac{1}{6} \frac{\Delta t}{\Delta x} \frac{\Delta t}{\Delta y} C^{+}|B| A^{+} \Delta_{x} Q_{i j k} \\
\widetilde{H}_{i j k} & :=\widetilde{H}_{i j k}+\frac{1}{6} \frac{\Delta t}{\Delta x} \frac{\Delta t}{\Delta y} C^{-}|B| A^{+} \Delta_{x} Q_{i j k} \\
\widetilde{H}_{i, j+1, k+1} & :=\widetilde{H}_{i+1, j, k+1}-\frac{1}{6} \frac{\Delta t}{\Delta x} \frac{\Delta t}{\Delta y} C^{+} B^{+} A^{+} \Delta_{x} Q_{i j k} \\
\widetilde{H}_{i, j+1, k} & :=\widetilde{H}_{i, j+1, k}-\frac{1}{6} \frac{\Delta t}{\Delta x} \frac{\Delta t}{\Delta y} C^{-} B^{+} A^{+} \Delta_{x} Q_{i j k} \\
\widetilde{H}_{i, j-1, k+1} & :=\widetilde{H}_{i, j-1, k+1}+\frac{1}{6} \frac{\Delta t}{\Delta x} \frac{\Delta t}{\Delta y} C^{+} B^{-} A^{+} \Delta_{x} Q_{i j k} \\
\widetilde{H}_{i, j-1, k} & :=\widetilde{H}_{i, j-1, k}+\frac{1}{6} \frac{\Delta t}{\Delta x} \frac{\Delta t}{\Delta y} C^{-} B^{-} A^{+} \Delta_{x} Q_{i j k} .
\end{aligned}
$$


Six additional updates are also required. These are obtained by replacing $i$ by $i-1$ in the fluxes above, and replacing $A^{+} \Delta_{x} Q_{i j k}$ by $A^{-} \Delta_{x} Q_{i j k}$. Note that the updates in (27) and (30) generalize the changes in $\widetilde{H}$ in (18). In order to approximate $B C A q_{x z y}$, similar updates are made to the $\widetilde{G}$ fluxes.

The $C^{+} B^{+} A^{+} \Delta_{x} Q_{i j k}$ term in (30) may be interpreted as follows. Each wave $\mathcal{W}_{i j k}^{p}$ in the expansion of $\Delta_{x} Q_{i j k}$ is split into waves moving in the $y$-direction as in(22). Then each $\beta^{p q} w^{q}$ is split into waves moving in the $z$-direction, i.e. $\beta^{p q} w^{q}=\sum_{l} \gamma^{p q l} s$. It is easily seen that

$$
C^{+} B^{+} A^{+} \Delta_{x} Q_{i j k}=\sum_{l} \sum_{q} \sum_{p}\left(\lambda^{p}\right)^{+}\left(\mu^{q}\right)^{+}\left(\nu^{l}\right)^{+} \gamma^{p q l} s^{l} .
$$

Regarding the implementation of these terms we do the same as done in (28) and (29).

The flux differences $A^{ \pm} \Delta_{x} Q_{i j k}$ are split in the $y$-direction accordingly. This yields the transverse wave strengths $\left(\delta^{p}\right)^{ \pm}$. The transverse flux difference $B^{+} A^{+} \Delta_{x} Q_{i j k}$ is defined as in (29). Note that this term already is computed when approximating the $B A Q_{x y}$ term. Next, split this flux difference into waves propagating in the $z$-direction, i.e. solve

$$
B^{+} A^{+} \Delta_{x} Q_{i j k}=\sum_{l} \eta^{l} s^{l}
$$

Using this, the update of $\widetilde{H}_{i, j+1, k+1}$ reads

$$
\widetilde{H}_{i, j+1, k+1}:=\widetilde{H}_{i, j+1, k+1}-\frac{1}{6} \frac{\Delta t}{\Delta x} \frac{\Delta t}{\Delta y} \sum_{l}\left(\nu^{l}\right)^{+} \eta^{l} s^{l} .
$$

In order to compute all updates in (30), also $B^{-} A^{+} \Delta_{x} Q_{i j k}$ and $B^{ \pm} A^{-} \Delta_{x} Q_{i j k}$ need to be split in the $z$-direction. Hence, in addition to the Riemann problems needed in approximating the second order cross-derivative terms like $B A q_{x y}$, four new Riemann problems are introduced per interface, by the third order terms.

The scheme obtained so far is the generalization of the "shift and average" scheme. The two-dimensional version is identical to the Corner Transport Upwind (CTU) scheme discussed by Colella [7]. The scheme is stable provided

$$
\max _{p, q, l}\left(\left|\lambda^{p}\right| \frac{\Delta t}{\Delta x},\left|\mu^{q}\right| \frac{\Delta t}{\Delta y},\left|\nu^{l}\right| \frac{\Delta t}{\Delta z}\right) \leq 1,
$$

but the scheme is only first order accurate on smooth solutions.

\subsection{The propagation of the correction wave}

In this section we look at the propagation of the correction wave, so that the order of accuracy increases. Note that the transverse propagation of the increment waves has already led to terms modeling cross-derivatives needed in a second-order Taylor series expansion. To obtain full second-order accuracy, it is thus formally necessary to only include the pure second-derivative terms $q_{x x}, q_{y y}$ and $q_{z z}$. This is accomplished by propagating the correction waves normal to the interfaces.

However, the resulting second-order accurate method would have very poor stability properties. In order to maintain good stability properties, we must also perform a transverse propagation of the correction waves in a multi-dimensional manner. This is developed in this section and then stability analysis is presented in Section 4. 


\subsubsection{Scalar equation}

We will illustrate the transverse and double transverse propagation of correction waves in the case of scalar advection. First the correction wave is added just as in the one-dimensional case, which gives the terms formally needed for second-order correction but ruins the stability. Again we present formulas only for the $x$-sweeps, with analogous modifications needed in the other directions.

$$
\widetilde{F}_{i j k}:=\widetilde{F}_{i j k}+\frac{1}{2} u\left(1-u \frac{\Delta t}{\Delta x}\right) \Delta_{x} Q_{i j k} .
$$

The flux (or slope) limiter is applied exactly as in the one-dimensional case, i.e. in (15) and (16), let $\mathcal{W}_{i}^{p}=\alpha_{i}^{p}=\Delta_{x} Q_{i j k}$.

In order to propagate the correction wave in the transverse direction, the natural approach is to move it in the same way as the increment wave (17). Let

$$
c(x, t)=\left(x-x_{i}+0.5 \Delta x-a\left(t-t_{n}\right)\right) \Delta_{x} Q_{i j} / \Delta x
$$

denote the correction wave associated with cell $C_{i-1, j k}$. (Note that a limited version of $\Delta_{x} Q_{i j k}$ could be used.) Then we have that

$$
\begin{aligned}
\widetilde{G}_{i, j+1, k} & :=\widetilde{G}_{i, j+1, k}-\frac{1}{\Delta t} \frac{1}{\Delta z} \frac{1}{\Delta x} \int_{t_{n}}^{t_{n+1}} \int_{z_{k}+w\left(t-t_{n}\right)}^{z_{k+1}} \int_{x_{i}}^{x_{i}+u\left(t-t_{n}\right)} v c(x, t) d x d z d t \\
& =\widetilde{G}_{i, j+1, k}+u v \frac{\Delta t}{\Delta x}\left(\frac{1}{4}-\frac{1}{6} u \frac{\Delta t}{\Delta x}\right) \Delta_{x} Q_{i j k} \\
& -u v w \frac{\Delta t}{\Delta z} \frac{\Delta t}{\Delta x}\left(\frac{1}{6}-\frac{1}{8} u \frac{\Delta t}{\Delta x}\right) \Delta_{x} Q_{i j k} .
\end{aligned}
$$

With corresponding updates of the $\widetilde{H}_{i, j+1, k+1}$ flux, stability is restored. However, the stability is not as good as wanted and the progation of the correction wave has to be done slightly differently in order to obtain (33). Instead of (35), the following update will be used:

$$
\begin{aligned}
\widetilde{G}_{i, j+1, k} & :=\widetilde{G}_{i, j+1, k}+\frac{1}{2} u v \frac{\Delta t}{\Delta x}\left(1-u \frac{\Delta t}{\Delta x}\right) \Delta_{x} Q_{i j k} \\
& -\frac{1}{2} u v w \frac{\Delta t}{\Delta x} \frac{\Delta t}{\Delta z}\left(1-u \frac{\Delta t}{\Delta x}\right) \Delta_{x} Q_{i j k} .
\end{aligned}
$$

Note that only the coefficients are changed. Even if the wave is not advected with the velocity field, it is possible to interprete the update as resulting from a wave propagation. The geometrical interpretation of the total motion of the correction wave is that it consists of several steps. In the first step, the wave is moved a distance $u \Delta t$ in the $x$-direction. This results in the familiar 1D update (34). Next, the wave is moved a distance $v \Delta t$ in the $y$-direction (transverse propagation), followed by a final step where the wave is moved a distance $w \Delta t$ in the $z$-direction (double transverse propagation). These three steps are shown in Figure 3. Note that also $\widetilde{G}$ and $\widetilde{H}$ fluxes in the downstream direction are updated. Since there are two coordinate directions covering the transverse propagation, the two last steps above have to be repeated but now in reversed order, i.e. first propagation in $z$ then in $y$. This will be discussed in more detail below. 


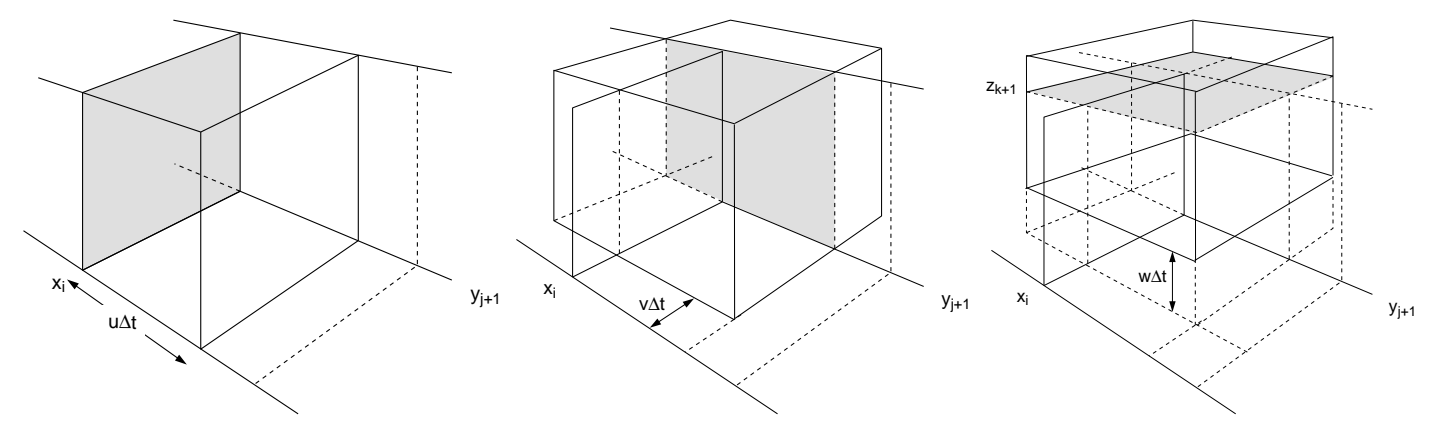

Figure 3: The propagation of the correction wave when the velocities are positive. The wave is first shifted a distance $u \Delta t$ in the $x$-direction, causing an update of $\widetilde{F}_{i j k}$. Next the wave is shifted a distance $v \Delta t$, causing an update of $\widetilde{G}_{i-1, j+1, k}$ and $\widetilde{G}_{i, j+1, k}$. Finally, the wave is advected a distance $w \Delta t$ in the $z$-direction, causing a update of four $\widetilde{H}$ fluxes.

\subsubsection{Linear systems}

As for the scalar example above, the algorithm for propagating the correction wave starts by updating the normal fluxes coordinate-wise,

$$
\widetilde{F}_{i j k}:=\widetilde{F}_{i j k}+\frac{1}{2} \sum_{p}\left|\lambda^{p}\right|\left(1-\frac{\Delta t}{\Delta x}\left|\lambda^{p}\right|\right) \widetilde{\mathcal{W}}_{i j k}^{p} .
$$

The limiting is done exactly as in 1D. This is a potential weakness of the method since then the limiter only takes into account waves propagating normal to the interfaces. At least for scalar problems there exist ways of doing multi-dimensional limiting, but they are more expensive in use (e.g., [1], [22], [34], and [48]). However, numerical experiments show that this simple one-dimensional limiting is able to control oscillations in a satisfactory way.

Naturally, the 3D-propagation of the correction wave has much in common with the propagation of the increment wave. Instead of propagating one wave at the time, a group of waves will be split into waves moving in the transverse direction.

Define

$$
\mathcal{S}=\sum_{p}\left|\lambda^{p}\right|\left(1-\frac{\Delta t}{\Delta x}\left|\lambda^{p}\right|\right) \widetilde{\mathcal{W}}_{i j k}^{p} .
$$

This term is to be split in both $y$ and $z$ directions. For example, consider the splitting in the $y$-direction of this correction term into an up-going and a down-going part, i.e., decompose $\mathcal{S}$ as

$$
\mathcal{S}=\sum_{q} \varepsilon_{i j k}^{q} w^{q}
$$

The wave $\varepsilon_{i j k}^{q} w^{q}$ will update two of the surrounding $\widetilde{G}$ fluxes depending on the sign of $\mu^{q}$. For example,

$$
\begin{aligned}
\widetilde{G}_{i, j+1, k} & =\widetilde{G}_{i, j+1, k}+\frac{1}{2} \frac{\Delta t}{\Delta x} \sum_{q}\left(\mu^{q}\right)^{+} \varepsilon_{i j k}^{q} w^{q}, \\
\widetilde{G}_{i-1, j+1, k} & =\widetilde{G}_{i-1, j+1, k}-\frac{1}{2} \frac{\Delta t}{\Delta x} \sum_{q}\left(\mu^{q}\right)^{+} \varepsilon_{i j k}^{q} w^{q} .
\end{aligned}
$$


Down-going waves would instead affect $\widetilde{G}_{i j k}$ and $\widetilde{G}_{i-1, j k}$. Recall that the flux updates made in the tranverse direction consist of the same terms as in the $2 \mathrm{D}$ scheme augmented by purely three-dimensional terms. In matrix notation, these two-dimensional updates are

$$
\begin{aligned}
\widetilde{G}_{i, j+1, k} & :=\widetilde{G}_{i, j+1, k}+\frac{1}{2} \frac{\Delta t}{\Delta x} B^{+}|A|\left(1-|A| \frac{\Delta t}{\Delta x}\right) \Delta_{x} Q_{i j k} \\
\widetilde{G}_{i j k} & :=\widetilde{G}_{i j k}+\frac{1}{2} \frac{\Delta t}{\Delta x} B^{-}|A|\left(1-|A| \frac{\Delta t}{\Delta x}\right) \Delta_{x} Q_{i j k} \\
\widetilde{G}_{i-1, j+1, k} & :=\widetilde{G}_{i-1, j+1, k}-\frac{1}{2} \frac{\Delta t}{\Delta x} B^{+}|A|\left(1-|A| \frac{\Delta t}{\Delta x}\right) \Delta_{x} Q_{i j k} \\
\widetilde{G}_{i-1, j k} & :=\widetilde{G}_{i-1, j k}-\frac{1}{2} \frac{\Delta t}{\Delta x} B^{-}|A|\left(1-|A| \frac{\Delta t}{\Delta x}\right) \Delta_{x} Q_{i j k}
\end{aligned}
$$

In two dimensions, it is possible to avoid the computation of the eigenvector expansion (or Riemann problem) in (39), by including $\mathcal{S}$ in the $2 \mathrm{D}$ part of the flux differences in (21). The proper modifications are

$$
\begin{aligned}
& A^{+} \Delta_{x} Q_{i j k}:=A^{+} \Delta_{x} Q_{i j k}-\mathcal{S} \\
& A^{-} \Delta_{x} Q_{i j k}:=A^{-} \Delta_{x} Q_{i j k}+\mathcal{S}
\end{aligned}
$$

Since $\frac{1}{2} \mathcal{S}$ already is computed in (37), including this transverse propagation requires virtually no extra work.

In two dimensions, the inclusion of the transverse propagation of the correction wave neither increases the order nor the stability limit of the method, but the accuracy may be improved. However, in three dimensions this contribution is essential.

In the three-dimensional case, similar updates as in (42) have to be made to the $\widetilde{H}$ fluxes. In matrix notation these terms are

$$
\begin{aligned}
\widetilde{H}_{i j, k+1} & :=\widetilde{H}_{i j, k+1}+\frac{1}{2} \frac{\Delta t}{\Delta x} C^{+}|A|\left(1-|A| \frac{\Delta t}{\Delta x}\right) \Delta_{x} Q_{i j k} \\
\widetilde{H}_{i j k} & :=\widetilde{H}_{i j k}+\frac{1}{2} \frac{\Delta t}{\Delta x} C^{-}|A|\left(1-|A| \frac{\Delta t}{\Delta x}\right) \Delta_{x} Q_{i j k} \\
\widetilde{H}_{i-1, j, k+1} & :=\widetilde{H}_{i-1, j, k+1}-\frac{1}{2} \frac{\Delta t}{\Delta x} C^{+}|A|\left(1-|A| \frac{\Delta t}{\Delta x}\right) \Delta_{x} Q_{i j k} \\
\widetilde{H}_{i-1, j k} & :=\widetilde{H}_{i-1, j k}-\frac{1}{2} \frac{\Delta t}{\Delta x} C^{-}|A|\left(1-|A| \frac{\Delta t}{\Delta x}\right) \Delta_{x} Q_{i j k} .
\end{aligned}
$$

In order to compute these updates, the correction term $\mathcal{S}$ has to be split in the $z$-direction.

Above we mentioned that there are good reasons for using updates of the form (36) instead of the more intuitive updates (35) resulting from pure advection of the correction wave. The best reason for this is that the stability is increased, cf. Section 4. The updates in (36) are also beneficial for accuracy reasons. Consider the Taylor expansion of $q$. Due to the large number of terms, we begin by including only terms that arise in the $2 \mathrm{D}$ case. Assume that we want to construct a third order accurate scheme. Then we have to approximate the following terms

$$
\begin{gathered}
-\frac{1}{6} \Delta t^{3}\left(A^{3} q_{x x x}+A^{2} B q_{y x x}+A B A q_{x y x}+A B^{2} q_{y y x}\right. \\
\left.+B A^{2} q_{x x y}+B A B q_{y x y}+B^{2} A q_{x y y}+B^{3} q_{y y y}\right) .
\end{gathered}
$$


In addition to these terms, we have to approximate terms arising in the truncation error in the approximations of the first and second order terms already derived.

For example, the truncation error in the approximation of $\frac{1}{2} \Delta t^{2} B A q_{x y}$ is dominated by

$$
-\frac{1}{4} \Delta t^{2} \Delta x\left(B^{+} A^{+}-B^{+} A^{-}+B^{-} A^{+}-B^{-} A^{-}\right) q_{x x y}=-\frac{1}{4} \Delta t^{2} \Delta x B|A| q_{x x y} .
$$

Then approximating

$$
\Delta t^{2} \Delta x B|A|\left(\frac{1}{4}-\frac{1}{6} \frac{\Delta t}{\Delta x}|A|\right) q_{x x y}
$$

will cancel the truncation error (46) and add the correct $q_{x x y}$ term from (45). Deriving similar expressions for the rest of the first and second order terms yields

$$
\begin{array}{r}
\frac{1}{6} \Delta t^{3} A B A q_{x y x}+\Delta t^{2} \Delta x B|A|\left(\frac{1}{4}-\frac{1}{6} \frac{\Delta t}{\Delta x}|A|\right) q_{x x y}+\Delta t^{2} \Delta x\left(\frac{1}{4}-\frac{1}{6} \frac{\Delta t}{\Delta x}|A|\right)|A| B q_{y x x} \\
-\frac{1}{6} \Delta t^{3} B A B q_{y x y}+\Delta t^{2} \Delta x A|B|\left(\frac{1}{4}-\frac{1}{6} \frac{\Delta t}{\Delta x}|B|\right) q_{y y x}+\Delta t^{2} \Delta x\left(\frac{1}{4}-\frac{1}{6} \frac{\Delta t}{\Delta x}|B|\right)|B| A q_{x y y} \\
+\frac{1}{6} \Delta t^{3} A^{3} q_{x x x}+\frac{1}{6} \Delta t^{3} B^{3} q_{y y y} .
\end{array}
$$

These terms have to be approximated in order to achieve a third order scheme. (Note that the update in (35) results in an approximation to only one of these terms, that shown in (47).)

Assume that $A$ and $B$ commute, i.e. that $A B=B A$. Then the expression above, with $q_{x x x}$ and $q_{y y y}$ omitted reduces to

$$
\frac{1}{2} \Delta t^{2} \Delta x B|A|\left(1-\frac{\Delta t}{\Delta x}|A|\right) q_{x x y} \quad+\quad \frac{1}{2} \Delta t^{2} \Delta y A|B|\left(1-\frac{\Delta t}{\Delta y}|B|\right) q_{y y x} .
$$

The transverse propagation of the correction wave as done in (42), yields exactly the desired approximation of the first term in (48). The second term is approximated by the similar transverse propagation of the correction wave emanating from interfaces in the $y$-direction.

For scalar advection with constant coefficients, a third order accurate scheme is easily obtained. Once transverse propagation of the correction waves is included, only the terms $q_{x x x}$ and $q_{y y y}$ need to be approximated, cf. [25].

Finally, consider the purely three-dimensional terms in the update, i.e. terms generalizing the last term in (36). Note that since this update of the $\widetilde{G}$ flux contains two $\Delta x$ and one $\Delta z$ and these fluxes are differenced in the $y$-direction, the contribution of this term approximates the fourth order derivative $q_{x x z y}$.

In a Taylor expansion of the scalar problem, all derivatives of fourth order involving two derivatives in $x$, and one in both $y$ and $z$, add up to

$$
-\frac{1}{2} \Delta t^{3} \Delta x u v w\left(1-\frac{\Delta t}{\Delta x} u\right) q_{x x y z}
$$

Above, we assumed that the matrices $A$ and $B$ commute, and we do the same here for $A, B$, and $C$. Hence, $(49)$ is split evenly between the $\widetilde{G}$ fluxes and the $\widetilde{H}$ fluxes in the system case, i.e.

$$
-\frac{1}{4} \Delta t^{3} \Delta x C B|A|\left(1-\frac{\Delta t}{\Delta x}|A|\right) q_{x x y z}-\frac{1}{4} \Delta t^{3} \Delta x B C|A|\left(1-\frac{\Delta t}{\Delta x}|A|\right) q_{x x z y} .
$$


The first term is approximated by the following flux updates

$$
\begin{aligned}
\widetilde{H}_{i j, k+1} & :=\widetilde{H}_{i j, k+1}-\frac{1}{4} \frac{\Delta t}{\Delta x} \frac{\Delta t}{\Delta y} C^{+}|B||A|\left(1-|A| \frac{\Delta t}{\Delta x}\right) \Delta_{x} Q_{i j k} \\
\widetilde{H}_{i j k} & :=\widetilde{H}_{i j k}-\frac{1}{4} \frac{\Delta t}{\Delta x} \frac{\Delta t}{\Delta y} C^{-}|B||A|\left(1-|A| \frac{\Delta t}{\Delta x}\right) \Delta_{x} Q_{i j k} \\
\widetilde{H}_{i, j+1, k+1} & :=\widetilde{H}_{i+1, j, k+1}+\frac{1}{4} \frac{\Delta t}{\Delta x} \frac{\Delta t}{\Delta y} C^{+} B^{+}|A|\left(1-|A| \frac{\Delta t}{\Delta x}\right) \Delta_{x} Q_{i j k} \\
\widetilde{H}_{i, j+1, k} & :=\widetilde{H}_{i, j+1, k}+\frac{1}{4} \frac{\Delta t}{\Delta x} \frac{\Delta t}{\Delta y} C^{-} B^{+}|A|\left(1-|A| \frac{\Delta t}{\Delta x}\right) \Delta_{x} Q_{i j k} \\
\widetilde{H}_{i, j-1, k+1} & :=\widetilde{H}_{i, j-1, k+1}-\frac{1}{4} \frac{\Delta t}{\Delta x} \frac{\Delta t}{\Delta y} C^{+} B^{-}|A|\left(1-|A| \frac{\Delta t}{\Delta x}\right) \Delta_{x} Q_{i j k} \\
\widetilde{H}_{i, j-1, k} & :=\widetilde{H}_{i, j-1, k}-\frac{1}{4} \frac{\Delta t}{\Delta x} \frac{\Delta t}{\Delta y} C^{-} B^{-}|A|\left(1-|A| \frac{\Delta t}{\Delta x}\right) \Delta_{x} Q_{i j k} .
\end{aligned}
$$

In addition to these updates, six additional updates are required in which $i$ is replaced by $i-1$ in the flux subscripts. The sign of the update is also switched.

Note that these updates involve the same interfaces as the double transverse propagation of the increment wave (30). The updates above require the solution of four additional Riemann problems. But due to the similarities, they may be included in the computation of the double transverse propagation of the increment wave. The proper updates for this inclusion are

$$
\begin{aligned}
B^{+} A^{+} \Delta_{x} Q_{i j k} & :=B^{+} A^{+} \Delta_{x} Q_{i j k}-\frac{3}{2} B^{+}|A|\left(1-|A| \frac{\Delta t}{\Delta x}\right) \Delta_{x} Q_{i j k} \\
B^{+} A^{-} \Delta_{x} Q_{i j k} & :=B^{+} A^{-} \Delta_{x} Q_{i j k}+\frac{3}{2} B^{+}|A|\left(1-|A| \frac{\Delta t}{\Delta x}\right) \Delta_{x} Q_{i j k} \\
B^{-} A^{+} \Delta_{x} Q_{i j k} & :=B^{-} A^{+} \Delta_{x} Q_{i j k}-\frac{3}{2} B^{-}|A|\left(1-|A| \frac{\Delta t}{\Delta x}\right) \Delta_{x} Q_{i j k} \\
B^{-} A^{-} \Delta_{x} Q_{i j k} & :=B^{-} A^{-} \Delta_{x} Q_{i j k}+\frac{3}{2} B^{-}|A|\left(1-|A| \frac{\Delta t}{\Delta x}\right) \Delta_{x} Q_{i j k}
\end{aligned}
$$

With these changes, both the double-transverse propagation of the increment wave and the correction wave are covered by the updates in (30). Finally, doing similar updates for the $\widetilde{G}$ flux will give approximations to the $q_{x x z y}$ term in (50).

Above, it was necessary to assume that the matrices commute when propagating the correction wave in the transverse direction. Note that this only affects (some) third and fourth order terms in a Taylor series. Not assuming this would require that a large number of new terms, like $A B A q_{x y x}$ had to be approximated. This would lead to an extremely complex code, and recall that we are interested in these terms for stability only. We have not been able to find examples in the system case for which the assumption of commuting matrices yields a more restrictive stability limit than the optimal we get for the scalar case.

\subsection{Non-linear problems and extensions}

So far, only linear problems have been considered. However, by the use of local linearizations, only minor changes have to be made in order to apply the scheme on non-linear problems (1). 
Motivated by the notation used for linear systems, the upwind contribution in (6) reads

$$
\begin{aligned}
\Delta_{i j k}^{u p} & =-\frac{\Delta t}{\Delta x}\left(\mathcal{A}^{+} \Delta_{x} Q_{i j k}+\mathcal{A}^{-} \Delta_{x} Q_{i+1, j k}\right)-\frac{\Delta t}{\Delta y}\left(\mathcal{B}^{+} \Delta_{y} Q_{i j k}+\mathcal{B}^{-} \Delta_{y} Q_{i, j+1, k}\right) \\
& -\frac{\Delta t}{\Delta x}\left(\mathcal{C}^{+} \Delta_{x} Q_{i j k}+\mathcal{C}^{-} \Delta_{z} Q_{i, j, k+1}\right) .
\end{aligned}
$$

Here, the terms $\mathcal{A}^{+} \Delta_{x} Q_{i j k}$, etc. are to be understood as symbols. The scheme is conservative provided

$$
\begin{aligned}
f\left(Q_{i j k}\right)-f\left(Q_{i-1, j k}\right) & =\mathcal{A}^{+} \Delta_{x} Q_{i j k}+\mathcal{A}^{-} \Delta_{x} Q_{i j k} \\
g\left(Q_{i j k}\right)-g\left(Q_{i, j-1, k}\right) & =\mathcal{B}^{+} \Delta_{y} Q_{i j k}+\mathcal{B}^{-} \Delta_{y} Q_{i j k} \\
h\left(Q_{i j k}\right)-h\left(Q_{i j, k-1}\right) & =\mathcal{C}^{+} \Delta_{z} Q_{i j k}+\mathcal{C}^{-} \Delta_{z} Q_{i j k}
\end{aligned}
$$

The process of finding the terms $\mathcal{A}^{+} \Delta_{x} Q_{i j k}$, etc., so that (54) is satisfied is a form of flux difference splitting. In addition to this, wave strengths $\mathcal{W}_{i j k}^{p}$ and wave velocities $\lambda_{i j k}^{p}$ have to be defined so that the flux terms $\widetilde{F}_{i j k}$ can be established. Note that waves and velocities are space dependent. There are $M_{w}$ waves involved, not necessarily equal to the dimension of the system $m$.

The flux difference splitting and associated waves and velocities may be obtained in different ways. The obvious one is to apply a local linearization that is conservative. A popular choice is the Roe solver [31], where a local approximation $A_{i j k}$ is made to the Jacobian $f^{\prime}(q)$. This involves finding the state $Q_{i j k}^{*}$ depending on $Q_{i-1, j k}$ and $Q_{i j k}$ so that if $A_{i j k}=f\left(Q_{i j k}^{*}\right)$ then

$$
A_{i j k}\left(Q_{i j k}-Q_{i-1, j k}\right)=f\left(Q_{i j k}\right)-f\left(Q_{i-1, j k}\right) .
$$

This property ensures conservation. The waves $\mathcal{W}_{i j k}^{p}$ and associated speeds $\lambda_{i j k}^{p}$ are derived exactly as in the linear case. A matrix satisfying (55) are found for several important systems like the Euler equations of gas dynamics.

Since the solution only consists of discontinuities, rarefaction waves may cause problems. If these waves are transonic, i.e. involve both positive and negative velocities, the numerical solution may contain physically incorrect shocks. In order to correct this, an entropy fix should be applied [16], [23], [33].

In the linear case, it is reasonable to compare wave strengths $\alpha_{i}^{p}$ (16) from neighboring interfaces, since the eigenvectors $r^{p}$ are constants. In the nonlinear case, the waves $\mathcal{W}_{i-1, j k}^{p}$, $\mathcal{W}_{i j k}^{p}$, and $\mathcal{W}_{i+1, j k}^{p}$ are not in general parallel, as vectors in $\mathbb{R}^{m}$. In CLAWPACK, the default limiting is based on a projection of $\mathcal{W}_{i \pm 1, j k}^{p}$ onto $\mathcal{W}_{i j k}^{p}$. The lengths of these projections are compared to the length of $\mathcal{W}_{i j k}^{p}$. The limiting is performed according to

$$
\begin{gathered}
\widetilde{\mathcal{W}}_{i j k}^{p}=\Phi\left(\theta_{i j k}^{p}\right) \mathcal{W}_{i j k}^{p}, \\
\theta_{i j k}^{p}= \begin{cases}\left(\mathcal{W}_{i-1, j k}^{p}, \mathcal{W}_{i j k}^{p}\right) /\left(\mathcal{W}_{i j k}^{p}, \mathcal{W}_{i j k}^{p}\right), & \lambda_{i j k}^{p}>0 \\
\left(\mathcal{W}_{i+1, j k}^{p}, \mathcal{W}_{i j k}^{p}\right) /\left(\mathcal{W}_{i j k}^{p}, \mathcal{W}_{i j k}^{p}\right), & \lambda_{i j k}^{p}<0\end{cases}
\end{gathered}
$$

where $(\cdot, \cdot)$ represents the inner product in $\mathbb{R}^{m}$. Note that this limiting generalizes the limiting used on linear systems, i.e. (15) and (16). 
We have used Roe-type Riemann solvers for the Euler equations in the computations presented here. There exist many other ways of defining the flux difference splitting and associated waves that can also be used in our formulation. In some situations it might be advantageous to use the exact solution of the Riemann problem. For example, when applied to the Euler equations, the Roe solver may produce negative pressure if the jump is large enough. Assume that $Q_{i j k}^{0}$, the exact solution for $x=x_{i}$, has been found. Then the flux splitting can be defined as

$$
\begin{aligned}
& \mathcal{A}^{+} \Delta_{x} Q_{i j k}=f\left(Q_{i j k}\right)-f\left(Q_{i j k}^{0}\right) \\
& \mathcal{A}^{-} \Delta_{x} Q_{i j k}=f\left(Q_{i j k}^{0}\right)-f\left(Q_{i-1, j k}\right) .
\end{aligned}
$$

The waves and the associated velocities needed for the correction waves also have to be specified. The states in the exact Riemann solution naturally define the jumps $\mathcal{W}_{i j k}^{p}$. If the original waves are discontinuities, the velocities should be chosen according to the Rankine-Hugoniot condition. If the waves are rarefaction waves, an average velocity could be used.

A third approach is worth considering; a hybrid version in which the exact solver defines the flux splitting, and the Roe solver defines the waves and velocities.

Transverse and double transverse propagation of increment and corrections waves are accomplished by constructing matrices $B_{i j k}$ and $C_{i j k}$. These matrices are simply taken as the Jacobians $g^{\prime}(q)$ and $h^{\prime}(q)$ respectively, evaluated at a state "close" to $Q_{i-1, j k}$ and $Q_{i j k}$. A good choice is to use the Roe-averaged state $Q_{i j k}^{*}$ already computed in the construction of the flux difference splitting.

Each product between a matrix and a vector corresponds to a Riemann problem. While an entropy fix may be necessary for the flux difference splitting, computations indicate that there is no need for this correction in the transverse direction. Hence, the Riemann problems used in computing these flux updates require less work than the Riemann problems in the normal direction. No entropy fix is needed, and the state used in evaluating the Jacobians is already computed.

A family of wave propagation schemes is defined by how the increment waves and correction waves are propagated. The order, accuracy and stability depends on how the waves are treated. It is useful to establish a notation refering to how the propagation is done. Let a specific scheme be defined by the three parameters $\left(m_{1}, m_{2}, m_{3}\right)$, where the integers $m_{1}, m_{2}, m_{3}$ have the following meaning:

$$
\begin{aligned}
& m_{1}= \begin{cases}1 & \begin{array}{l}
\text { The second order correction wave is not included } \\
\text { thus the method is formally first order accurate }
\end{array} \\
2 & \text { The correction wave is included }\end{cases} \\
& m_{2}= \begin{cases}0 & \text { No transverse propagation. } \\
1 & \text { Transverse propagation of the increment wave. } \\
2 & \text { Transverse propagation of both increment and } \\
& \text { correction wave. (Requires } \left.m_{1}=2 .\right)\end{cases} \\
& m_{3}= \begin{cases}0 & \text { No double transverse propagation. } \\
1 & \begin{array}{l}
\text { Double transverse propagation of the increment } \\
\text { wave. (Requires } \left.m_{2}>0 .\right)
\end{array} \\
2 & \begin{array}{l}
\text { Double transverse propagation of both increment and } \\
\text { correction wave. (Requires } \left.m_{2}=2 .\right)
\end{array}\end{cases}
\end{aligned}
$$


For example, Method $(1,1,0)$ defines the first order scheme in which the increment wave moves as in 2D. The complete wave propagation method is Method $(2,2,2)$. (In CLAWPACK, $m_{2}$ and $m_{3}$ are specified by a two digit integer, hence the method is identified by only two parameters $m_{1}$ and $\left.10 \cdot m_{2}+m_{3}.\right)$

The number of Riemann problems needed increases as the propagation gets more and more complex. In the first order donor-cell method, i.e. Method $(1,0,0)$, only one Riemann problem has to be solved per interface. If the increment wave moves as in the $2 \mathrm{D}$ case (Method $(1,1,0)$ ), four additional problems have to be solved. The generalization of the "shift and average" scheme, i.e. Method $(1,1,1)$ requires a total of 13 Riemann problems. Five Riemann problems have to be solved per interface for (the unconditionally unstable) Method $(2,1,0)$. The simple generalization of the full $2 \mathrm{D}$ wave propagation scheme, Method $(2,2,0)$, requires seven Riemann problems. This number may be reduced to five as noted above. The shift and average scheme plus propagation normal to the interface of the correction wave (Method $(2,1,1)$ ), requires the solution of 15 Riemann problems per interface. The same number is required for Method $(2,2,2)$, the full three-dimensional wave propagation scheme.

The total number of Riemann problems needed per cell is three times the number per interface. This indicates that the $3 \mathrm{D}$ wave propagation methods may be computationally expensive, depending on the choice of Riemann solver. Using the Roe-solver approach, only one of the Riemann problems, per interface, requires the entropy fix and computation of the Roe-average state. The rest of the Riemann problems involve significantly less work, essentially just a matrix-vector multiply to decompose a vector into eigencomponents.

The advantage of using (6) with the flux difference splitting (54) in the definition of the upwind term $\Delta_{i j k}^{u p}$, is that the flux functions are not explicitly needed. As a concequence, the same scheme is applicable to a larger class of quasilinear hyperbolic problems of the form (3). The matrices may depend on $x, y, z$, and $t$ in addition to the solution $q$. In the "flux difference splitting" (which is called "fluctuation splitting" more generally in [26]), the waves, and associated velocities are easily defined. However, note that spacially-varying matrices may result in reflections in addition to transmission of waves across cell interfaces. This complicates the problem, but may still be treated within the same framework, cf. [26] for details.

\section{Applications}

In this section we consider the Euler equations which models an inviscid, compressible and non-heat conducting gas. The system may be written as

$$
\left[\begin{array}{c}
\rho \\
\rho u \\
\rho v \\
\rho w \\
E
\end{array}\right]_{t}+\left[\begin{array}{c}
\rho u \\
\rho u^{2}+p \\
\rho u v \\
\rho u w \\
u(E+p)
\end{array}\right]_{x}+\left[\begin{array}{c}
\rho v \\
\rho u v \\
\rho v^{2}+p \\
\rho v w \\
v(E+p)
\end{array}\right]_{y}+\left[\begin{array}{c}
\rho w \\
\rho u w \\
\rho v w \\
\rho w^{2}+p \\
w(E+p)
\end{array}\right]_{z}=0
$$

where $\rho$ and $p$ denote the density and pressure. The velocity components in the $x, y$, and $z$ directions are $u, v$, and $w$, respectively. If we assume that the gas is polytropic, the total energy density $E$ is

$$
E=\frac{1}{2} \rho\left(u^{2}+v^{2}+w^{2}\right)+\frac{1}{\gamma-1} p
$$


where $\gamma$ is a gas constant taken to be 1.4 in the examples below.

The Roe approximate Riemann solver is used in all the following examples. Thus, the Jacobian matrices are evaluated at a specific state, as described in Section 2, in order to ensure conservation and to define the waves needed in the algorithm. For details on this see [31] and the Riemann solver routines in the CLAWPACK software [27]. In [26], the details concerning the solution of the two-dimensional isothermal equations are given. We refer to that paper, since the main principles are the same, when it comes to defining matrices and flux splitting, as in the three-dimensional full Euler case.

\subsection{Smooth Euler solution}

In order to verify second order convergence, we consider an initial value problem with a smooth solution, at least for the time interval considered. Initially, the gas is at rest and

$$
\rho(x, y, z, 0)=E(x, y, z, 0)=1+0.1 e^{-30(r-1)^{2}},
$$

where $r=\sqrt{x^{2}+y^{2}+z^{2}}$. The solution will remain spherically symmetric. Due to this, it is possible to formulate (56) as a one-dimensional conservation law with a source term,

$$
\left[\begin{array}{c}
\rho \\
\rho u \\
E
\end{array}\right]_{t}+\left[\begin{array}{c}
\rho u \\
\rho u^{2}+p \\
u(E+p)
\end{array}\right]_{r}=-\frac{1}{r}\left[\begin{array}{c}
\rho u \\
\rho u^{2} \\
u(E+p)
\end{array}\right] .
$$

Here, $u$ denotes the radial velocity. An accurate reference solution is obtained by solving this equation using one-dimensional CLAWPACK routines. Due to the symmetry, the computational domain for the three-dimensional scheme can be taken to be a single octant $(x, y, z) \in[0,2] \times$ $[0,2] \times[0,2]$. At the boundaries $x=0, y=0$, and $z=0$, symmetric boundary conditions are used. The remaining boundaries are all of the outflow type described in [26].

Boundary conditions are imposed in exactly the same way as described in [26] for one and two dimensions: two additional rows of "ghost cells" along each edge are introduced. Values in the ghost cells are set in each time step in such a way as to give the correct behavior. Zeroorder extrapolation is used at the outflow boundaries while reflection is used at the symmetry planes.

The solution is computed on an $N \times N \times N$ grid at time $t=0.5$. In Table 1 , the errors for Method $(1,1,1)$ are given for $N=20,40$ and 80 . The errors are computed by comparing with the one-dimensional reference solution. The rate of convergence is estimated using the two finest grids according to the formula

$$
\text { convergence order }=\frac{1}{\ln 2} \ln \left(\frac{\operatorname{error}(\Delta x)}{\operatorname{error}(\Delta x / 2)}\right) .
$$

The errors in $\rho v$ and $\rho w$ equal the errors in $\rho u$. As expected, this scheme is approximately first order accurate. In Table 2, the errors for the full wave propagation scheme Method $(2,2,2)$ are given. Since the solution is smooth, no limiting is performed. According to the figures in this table, the scheme is second order accurate on this problem. In Figure 4, scatter plots of the density using $N=20$ and $N=40$ are shown. Every value in the $N \times N \times N$ grid is plotted against $r$, the distance from the origin. The solid curve corresponds to the reference solution. In the computations for both the first and second order scheme, fixed time steps are used giving a Courant number of approximately 0.78 . 


\begin{tabular}{|c|ccc|ccc|}
\hline & \multicolumn{3}{|c|}{ 1-norm errors } & \multicolumn{3}{c|}{ max-norm errors } \\
\hline$N$ & $\rho$ & $\rho u$ & $E$ & $\rho$ & $\rho u$ & $E$ \\
\hline 20 & $6.482 \cdot 10^{-3}$ & $2.498 \cdot 10^{-3}$ & $9.425 \cdot 10^{-3}$ & $9.398 \cdot 10^{-3}$ & $7.496 \cdot 10^{-3}$ & $1.373 \cdot 10^{-2}$ \\
\hline 40 & $3.492 \cdot 10^{-3}$ & $1.338 \cdot 10^{-3}$ & $5.074 \cdot 10^{-3}$ & $5.292 \cdot 10^{-3}$ & $4.389 \cdot 10^{-3}$ & $7.738 \cdot 10^{-3}$ \\
\hline 80 & $1.831 \cdot 10^{-3}$ & $7.003 \cdot 10^{-4}$ & $2.658 \cdot 10^{-3}$ & $2.729 \cdot 10^{-3}$ & $2.350 \cdot 10^{-3}$ & $3.984 \cdot 10^{-3}$ \\
\hline \hline order & 0.93 & 0.93 & 0.93 & 0.96 & 0.90 & 0.96 \\
\hline
\end{tabular}

Table 1: Errors for the smooth Euler problem computed with Method $(1,1,1)$ on a $N \times N \times N$ grid. The errors in $\rho v$ and $\rho w$ equal the errors in $\rho u$. The convergence order is estimated on the two finest grids.

\begin{tabular}{|c|ccc|ccc|}
\hline & \multicolumn{3}{|c|}{ 1-norm errors } & \multicolumn{3}{c|}{ max-norm errors } \\
\hline$N$ & $\rho$ & $\rho u$ & $E$ & $\rho$ & $\rho u$ & $E$ \\
\hline 20 & $4.334 \cdot 10^{-3}$ & $1.705 \cdot 10^{-3}$ & $6.290 \cdot 10^{-3}$ & $6.985 \cdot 10^{-3}$ & $5.140 \cdot 10^{-3}$ & $1.012 \cdot 10^{-2}$ \\
\hline 40 & $1.361 \cdot 10^{-3}$ & $4.767 \cdot 10^{-4}$ & $1.961 \cdot 10^{-3}$ & $2.331 \cdot 10^{-3}$ & $1.761 \cdot 10^{-3}$ & $3.368 \cdot 10^{-3}$ \\
\hline 80 & $3.541 \cdot 10^{-4}$ & $1.254 \cdot 10^{-4}$ & $5.091 \cdot 10^{-4}$ & $6.387 \cdot 10^{-4}$ & $4.931 \cdot 10^{-4}$ & $9.216 \cdot 10^{-4}$ \\
\hline \hline order & 1.94 & 1.93 & 1.95 & 1.87 & 1.84 & 1.87 \\
\hline
\end{tabular}

Table 2: Errors for the smooth Euler problem computed with Method $(2,2,2)$ on a $N \times N \times N$ grid. The errors in $\rho v$ and $\rho w$ equal the errors in $\rho u$. The convergence order is estimated on the two finest grids.
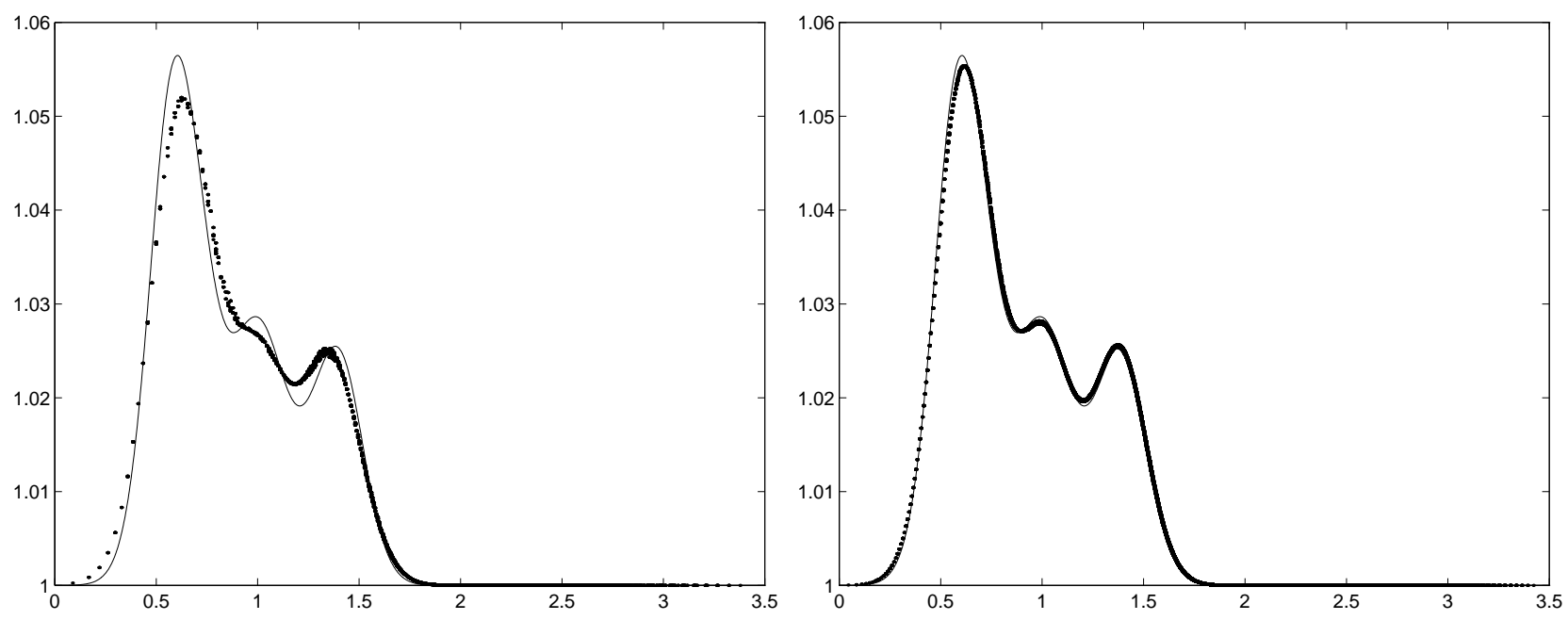

Figure 4: (Example of Section 3.1) Scatter plots for the Euler equation with smooth solution, computed with Method $(2,2,2)$. The density is depicted against the distance from origin. In the left picture, $N=20$, while in the right picture $N=40$. The solid curve is the reference fine-grid solution from a one-dimensional spherically-symmetric calculation. 


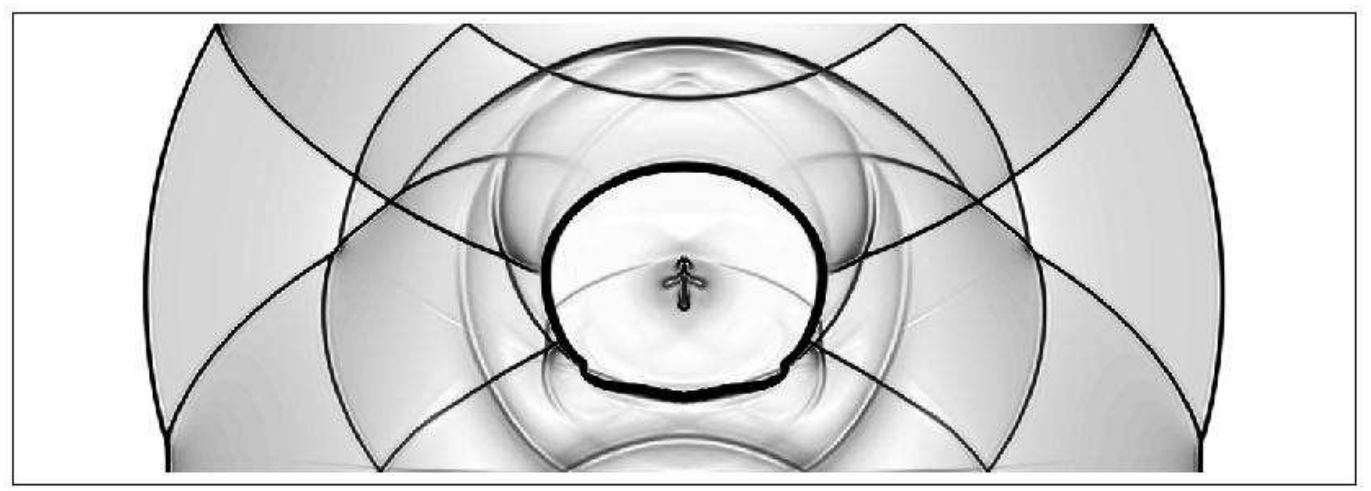

Figure 5: (Example of Section 3.2) A schlieren type image showing the density at $t=0.7$. The two-dimensional axisymmetric problem has been solved using a $600 \times 400$ grid on half the domain $(0 \leq x \leq 1.5,0 \leq y \leq 1)$ and reflected to $-1.5 \leq x \leq 0$.

\subsection{A spherical Riemann problem}

In this example we consider a spherical Riemann problem between two parallel walls at $z=0$ and $z=1$. Initially the gas is at rest with density and pressure $\rho_{\text {out }}=1$ and $p_{\text {out }}=1$ everywhere except in a sphere centered at $(0,0,0.4)$ with radius 0.2 . Inside the sphere $\rho_{i n}=1$ and $p_{i n}=5$. The jump in pressure results in a strong outward moving shock wave and contact discontinuity, and an inward moving rarefaction wave. This inward moving wave causes a local "implosion", and a second outward moving shock wave is created. The main features of the solution are the interactions between these waves and between waves and the walls. Another significant feature is the development of a near stationary low density region in the center of the domain. Until the initial shock wave reaches the lower wall, the solution is spherically symmetric. After this, the solution will remain cylindrically symmetric. Hence, it is possible to formulate this as a two-dimensional problem with a source term,

$$
\left[\begin{array}{c}
\rho \\
\rho u \\
\rho w \\
E
\end{array}\right]_{t}+\left[\begin{array}{c}
\rho u \\
\rho u^{2}+p \\
\rho u w \\
u(E+p)
\end{array}\right]_{r}+\left[\begin{array}{c}
\rho w \\
\rho u w \\
\rho w^{2}+p \\
w(E+p)
\end{array}\right]_{z}=-\frac{1}{r}\left[\begin{array}{c}
\rho u \\
\rho u^{2} \\
\rho u w \\
u(E+p)
\end{array}\right]
$$

where $u$ now denotes the radial velocity in the $x-y$ plane. This equation is solved using twodimensional CLAWPACK routines, and the results are used for comparison. The two-dimensional problem is solved on a $600 \times 400$ grid. Due to the symmetry, the computational domain is $(r, z)=[0,1.5] \times[0,1]$, where $r=\sqrt{x^{2}+y^{2}}$. Figure 5 shows a schlieren picture of the solution at $t=0.7$. This picture emulates a photographic technique used in physical experiments. The magnitude of the density gradient is depicted. The larger the gradient, the darker is the region. Strong nonlinear shading is used in order to enhance weak structures in the flow. In this figure, also the symmetric part is depicted. Note the strong contact discontinuity surrounding the low density region near the center. There is also an unphysical feature visible near the center of the domain. It is caused by the wave focusing and is highly grid dependent. Due to the nonlinear shading in this schlieren image, the magnitude of this feature is exaggerated. Its influence on the rest of the flow field is actually very weak. 

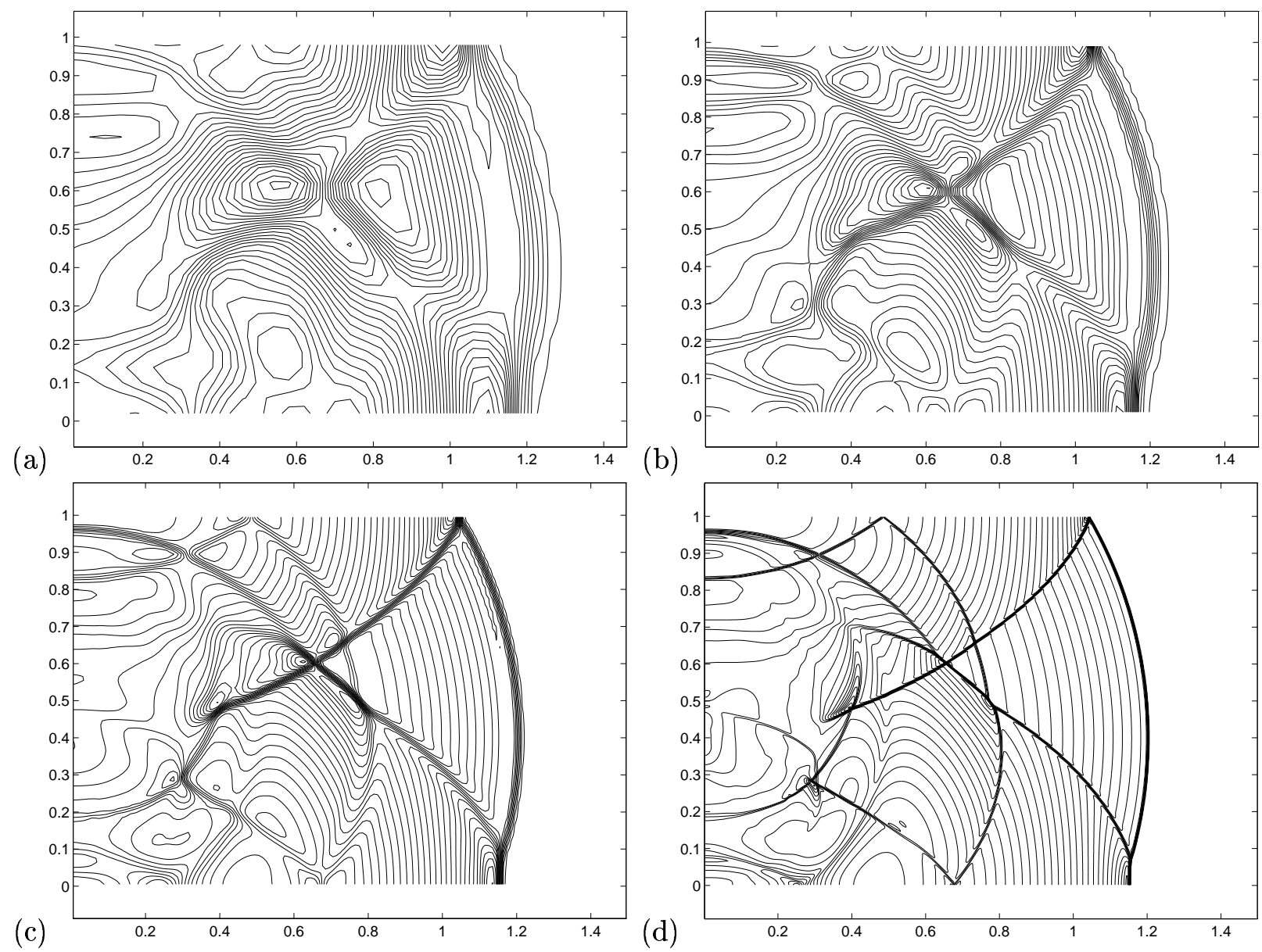

Figure 6: (Example of Section 3.2) The pressure in the $x-z$ plane at $t=0.7$ computed on different grids: (a) $37 \times 37 \times 25$, (b) $75 \times 75 \times 50$, (c) $150 \times 150 \times 100$. In (d), results from the corresponding $2 \mathrm{D}$ computation using a $600 \times 400$ grid is shown.

Due to the symmetry, the computational domain for the three-dimensional algorithm is $(x, y, z) \in[0,1.5] \times[0,1.5] \times[0,1]$. In Figure 6 , the pressure in the $x$ - $z$ plane at $t=0.7$ is shown using an increasing number of cells. The solution is computed using Method $(2,2,2)$. The two-dimensional results are also given for comparison.

Another way of graphically indicating convergence is to consider scatter plots, like those used in the previous example. In Figure 7, the pressure is plotted against the distance from the $z$-axis. The plane used is $z=0.4$ at $t=0.7$. These pictures indicate that not only are the main features well resolved, but also the finer structures appear to be converging. In both the two-dimensional and the three-dimensional computations MC-limiters are used on all waves. In these computations the Courant number was approximately 0.9 .

Additional images and animations from both the 2D and 3D computations may be found on the Web page http://www.amath. washington.edu/ ${ }^{\sim} \mathrm{rjl}$ l/clawpack/3dpaper.html 

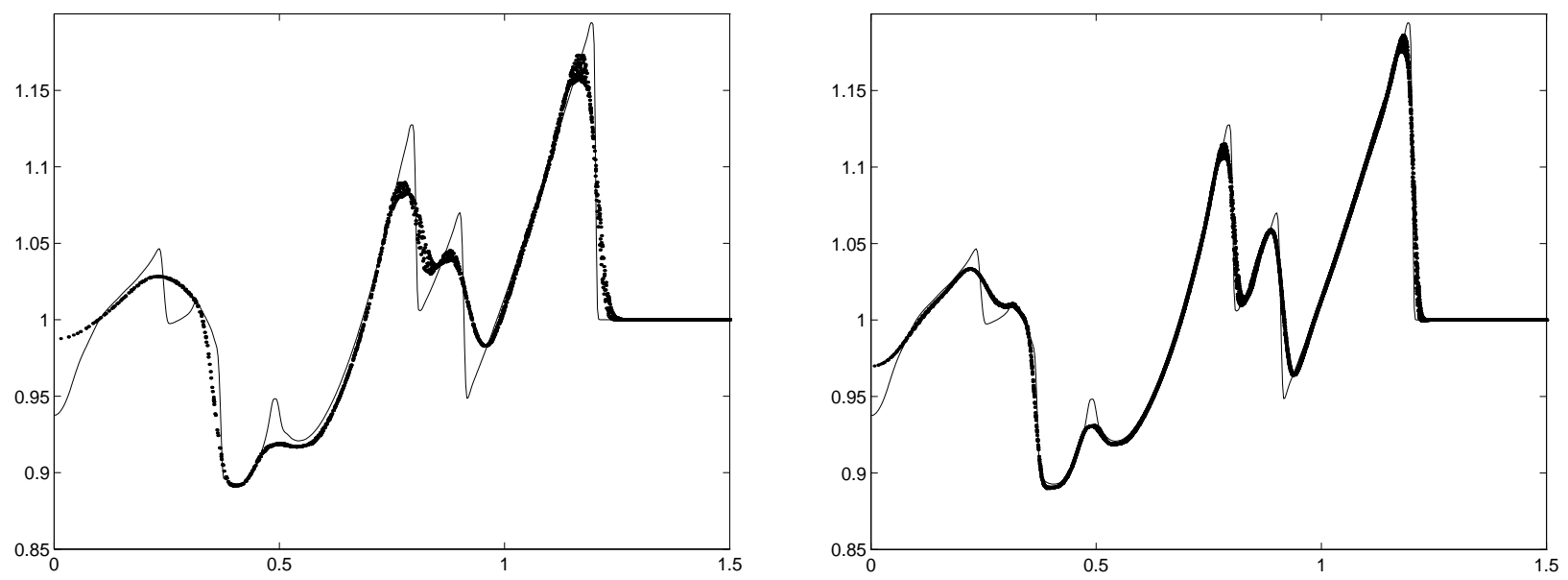

Figure 7: (Example of Section 3.2) Scatter plots of the pressure versus the distance from the $z$-axis in the plane $z=0.4$. In the left picture the computational grid is $75 \times 75 \times 50$, while in the right a $150 \times 150 \times 50$ grid is used. The solid curve corresponds to the $600 \times 4002 \mathrm{D}$ computation.

\subsection{Vorticity generated by a shock wave}

As an example of a pure three-dimensional problem, we consider the situation where shocks interact with variable density regions. Similar problems have been considered for the study of shocks propagating through inhomogeneous media. One such example is taken from the design of SCRAM-jets, where shock waves may help to mix fuel and air by generating vorticity. The problem is simplified by considering a single planar wave hitting a cylinder or sphere filled with fuel, cf. [10], [28]. In these papers a two component gas is considered. Here, we only consider a single component gas.

Initially, the gas is at rest. The pressure and the density equal unity everywhere, except for two cylinders perpendicular to each other. Both cylinders contain constant state gas. The radius of each cylinder is $r=0.2$. In the cylinder along the $z$-axis, i.e. with symmetry axis $x=y=0$, the density is $\rho=1$ but the pressure is $p=10$, and thus a cylindrical shock wave will emanate from the pressure jump at the surface of this cylinder. The other cylinder is parallel to the $y$-axis, with symmetry axis $x=0.4$ and $z=0$. In the latter cylinder, the the pressure is $p=1$ but the density is lower, $\rho=0.1$. This contact discontinuty is stationary until it is disturbed by the shock waves. The initial set up is depicted in Figure 8(a).

The experiment is set up in such a way that as the front shock hits the low density cylinder, a huge amount of vorticity is produced. Let $\vec{\omega}=\nabla \times \vec{u}$ denote the vorticity. The vorticity equation reads

$$
\frac{\partial \vec{\omega}}{\partial t}=\nabla \times(\nabla \times \vec{\omega})+\frac{\nabla \rho \times \nabla p}{\rho^{2}} .
$$

The last term on the right hand side is called the baroclinic source term, and is responsible for the vorticity production.

The computational domain is $[0,1.5] \times[0,1] \times[0,0.5]$. Symmetry across the plane $z=0$ is assumed, though this is not physically correct for such a problem due to the turbulent behaviour. The computation is performed on a $300 \times 200 \times 100$ grid with Method $(2,2,2)$, and the MC-limiter. 
As the incident shock wave hits the cylinder, it separates into a reflected smooth wave and a penetrating shock wave. Due to the higher sound speed inside the cylinder, the latter wave will speed up. As the shock wave runs through the cylinder, the latter collapses leaving a major counter-clockwise rotating vortex, with smaller vortex tubes on the rim. The dynamics are extremely complex as the vortex tubes rotate around each other, get twisted, stretched and burst.

Figure 8 shows volume rendering of the absolute value of the density gradient for three $t$-values, i.e. the same quantity as displayed in a schlieren image. It is especially useful to study this quantity in the context of shock problems, since the density jumps across both shock waves and contact discontinuities.

At $t=0.1$, Figure $8(\mathrm{~b})$, the incident shock wave has partially encapsulated parts of the low density cylinder. The two essentially cylindrical surfaces are, from the left, the contact surface and the shock wave. Note that this part of the shock wave has a lower speed than the one encapsulating the low density cylinder. As mentioned above, this is due to the increased sound speed inside this cylinder. Also note that the low density cylinder has started to collapse due to the interaction with the shock wave. In Figure $8(\mathrm{c})$, at $t=0.3$, the roll-up of the low density cylinder gives rise to a major vortex. Note that the incident shock wave has passed this vortex in most of the computational domain, and is connected to the vortex by a triple shock configuration. In Figure $8(\mathrm{~d})$, the time is $t=0.5$, and the incident shock has started to leave the computational domain. To the left, the near cylindrically shaped contact discontinuity dominates. Note the "fingers" on the rim of the main vortex tube. Inside each of these fingershaped regions, there is a loop of smaller vortex tubes. This is illustrated more clearly at a later time $t=0.8$ in Figure 9, where two different quantites are visualized together as described below. These vortex loops are advected by the the main vortex. The weak shock located at the top of the main vortex is an implosion shock, similar to the one that appeared in the previous example.

The images in Figure 8 were produced using the visualization tool Viz [49], freely available for non-commersial use. Viz is a highly interactive tool for displaying large voxel-based data sets. It was initially created to utilize the potential of hardware accelerated 3D textures. The voxel is the 3D generalization of the pixel, including both a color value and a opacity value. Due to the possibilities of adding opacity to the dataset, a range of values can be studied, in contrast to the more common iso-surface technique. So even though the images in Figure 8 appear to be iso-surfaces, they are not. Both stronger and weaker discontinuities are displayed, the latter appear more transparent than the former, cf. Figure 8(d).

In Figure 9, another application of voxels graphics and Viz is shown. The time is $t=0.8$ and only a subset of the data is shown, centered along the main vortex. Two scalar fields are displayed in the same scene. In order to visualize the smaller vortex tubes on the rim of the main vortex, the enstrophy is used, i.e. $|\vec{\omega}|^{2}$. Note that the surface of these tubes are made darker to enhance the shape of these features. The other quantity shown is the dominating low pressure region. In contrast to the enstrophy, the surface of this region is made lighter. Animations and additional images from this computation may be found on the Web page

http://www . amath. washington.edu/ ${ }^{\sim} \mathrm{jjl} / \mathrm{clawpack/3dpaper.html}$ 

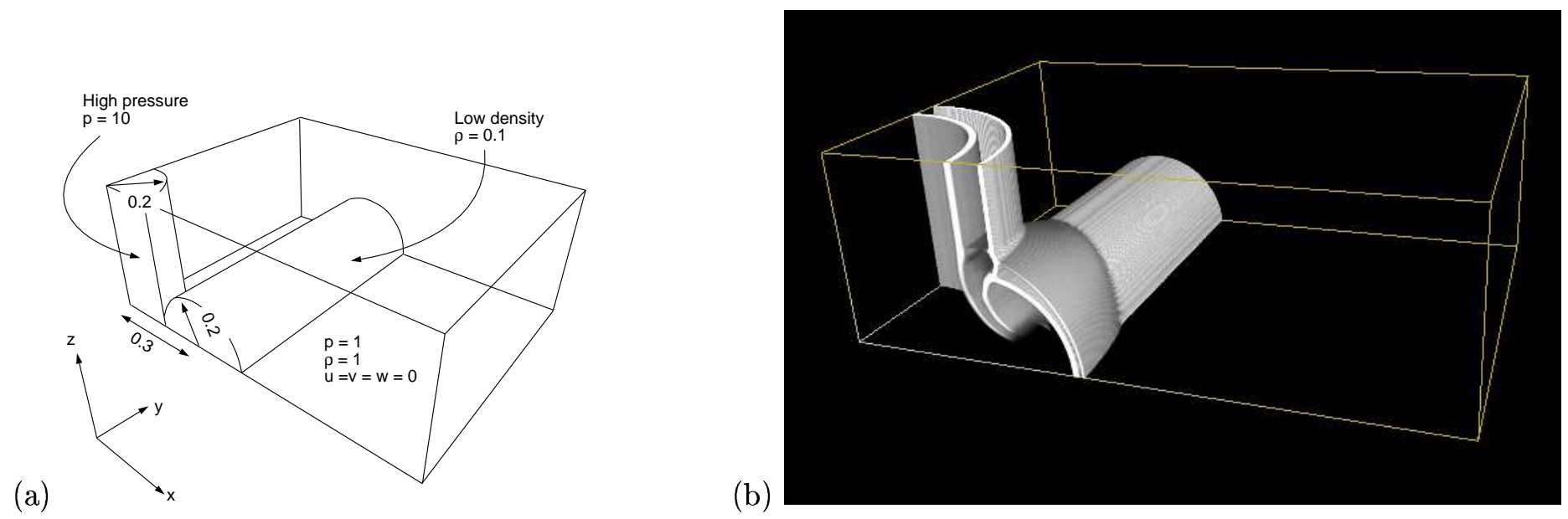

(c)
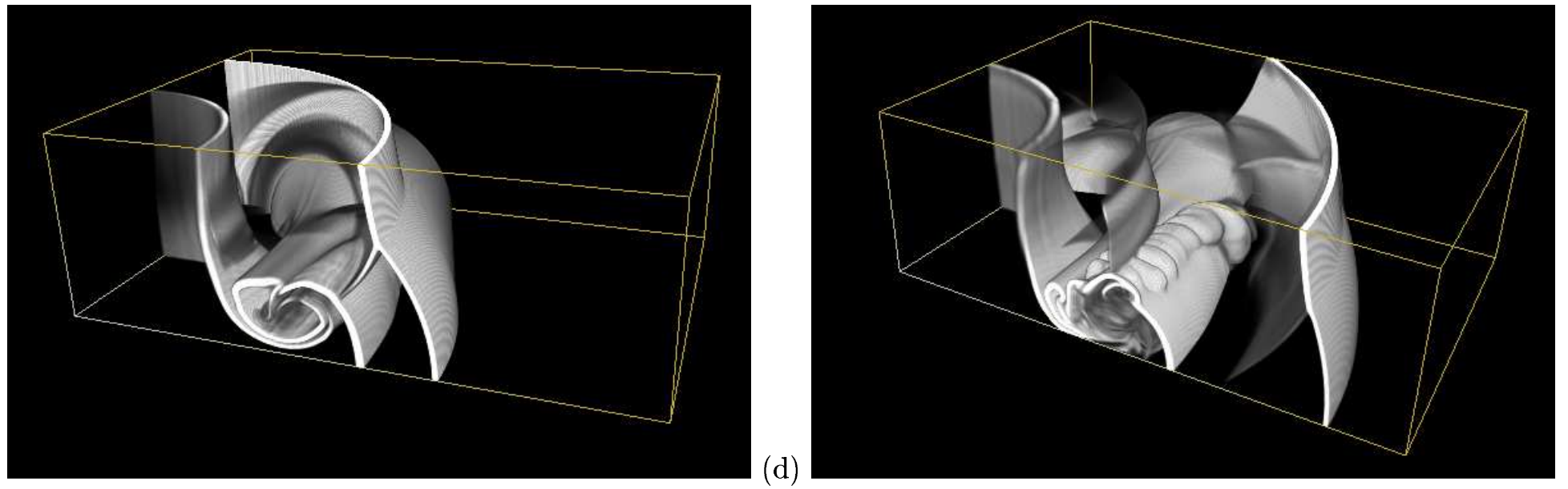

Figure 8: (Example of Section 3.3) (a) The initial condition. The rest of the images depict the quantity $|\nabla \rho|$, also used in schlieren pictures, at different time: (b) $t=0.1$, (c) $t=0.3$, (d) $t=0.5$. 


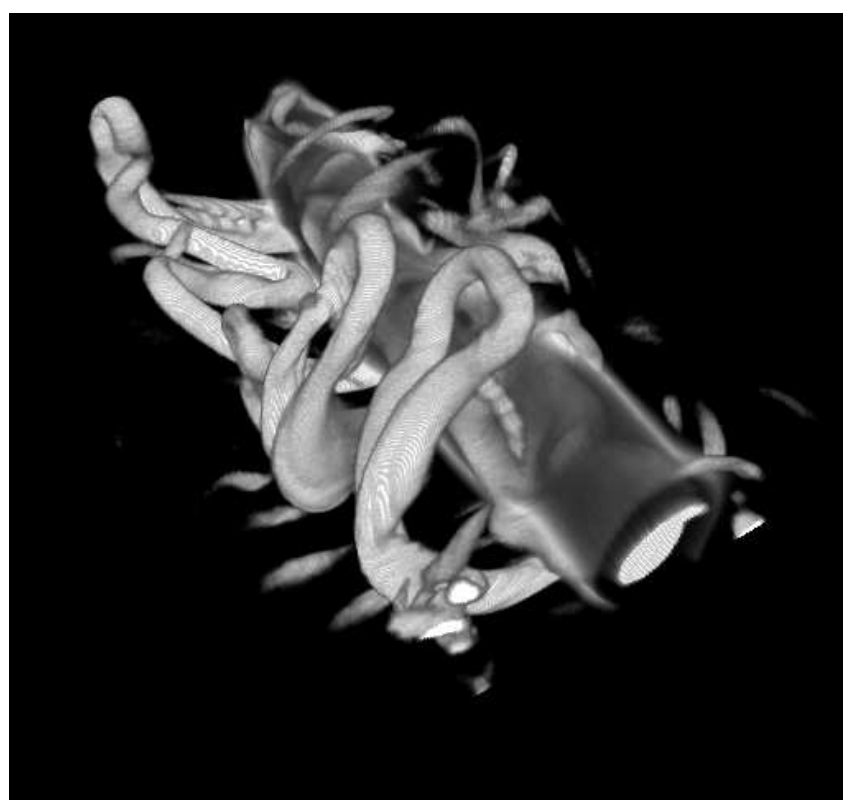

Figure 9: (Example of Section 3.3) A subset of the computational domain shown at $t=0.8$. Both enstrophy and (low) pressure are visualized in this picture (cf. text).

\section{Stability}

In this section we investigate the stability properties of the algorithms introduced in Section 2 when applied to linear advection and acoustics problems. Initially we study the case with no limiter, so that the numerical scheme itself is linear and we can use von Neumann analysis. In Section 4.5 we also investigate the effect of limiters by running the algorithm and calculating amplification factors for different sets of initial data. This is of interest because we have found that some methods which are unstable without limiters due to exponential growth of high frequency components are stabilized by the use of limiters.

We consider two linear problems, first the scalar advection equation with constant coefficients in Section 4.1 and then the linearized Euler equations in Section 4.4. We find that the algorithms have similar stability properties in each case.

For a general linear system $q_{t}+A q_{x}+B q_{y}+C q_{z}=0$ in which the matrices $A, B, C$ have eigenvalues $\lambda^{p}, \mu^{q}$, and $\nu^{l}$, respectively, we define the directional Courant numbers as

$$
\omega_{1}=\frac{\Delta t}{\Delta x} \max _{p}\left|\lambda^{p}\right|, \quad \omega_{2}=\frac{\Delta t}{\Delta y} \max _{q}\left|\mu^{q}\right|, \quad \omega_{3}=\frac{\Delta t}{\Delta z} \max _{l}\left|\nu^{l}\right|,
$$

and set $\vec{\omega}=\left(\omega_{1}, \omega_{2}, \omega_{3}\right)$, not to be confused with the vorticity in the previous section. The Courant number is the maximum of these three quantites. The best we can hope for with methods of the type developed here is that they will be stable for all Courant numbers up to 1 , i.e., for

$$
\max \left(\omega_{1}, \omega_{2}, \omega_{3}\right) \leq 1
$$

This set is a cube in $\omega_{1}-\omega_{2}-\omega_{3}$ space. For most methods the stability restriction is most severe along the diagonal of the cube, i.e., for the special case where $\omega_{1}=\omega_{2}=\omega_{3}$, and it is often 
possible to obtain analytic expressions for the amplification factor in this special case. When this case is considered we will denote the common value of $\vec{\omega}$ by $\omega_{d}$,

$$
\omega_{d} \equiv \omega_{1}=\omega_{2}=\omega_{3}, \quad \text { along diagonal. }
$$

Von Neumann analysis is based on inserting the Fourier mode

$$
Q_{I J K}=e^{i(\xi I+\eta J+\theta K)},
$$

into the numerical scheme. We use capital letters $I J K$ as the grid index here to avoid confusion with $i=\sqrt{-1}$. For a linear method on a linear problem, this will result in an expression of the form

$$
\bar{Q}_{I J K}=T(\xi, \eta, \theta, \vec{\omega}) Q_{I J K},
$$

where $\bar{Q}$ denotes the numerical solution at the next time step. For scalar advection $T$ is a scalar, while for a system of $m$ equations $T$ is an $m \times m$ matrix. In the scalar case, the method is stable for given $\vec{\omega}$ if

$$
\mathcal{T}(\vec{\omega}) \equiv \max _{\xi, \eta, \theta}|T(\xi, \eta, \theta, \vec{\omega})| \leq 1,
$$

where maximum is taken for $-\pi \leq \xi, \eta, \theta \leq \pi$. In fact for all the methods considered, $T(0,0,0, \vec{\omega})=1$ for all $\vec{\omega}$, so (64) will be satisfied with equality in the stability region.

Only in simple cases is it possible to obtain analytic expressions for $\mathcal{T}(\vec{\omega})$. The diagonal case where $\vec{\omega}=\vec{\omega}_{d} \equiv\left(\omega_{d}, \omega_{d}, \omega_{d}\right)$ will be denoted by $\mathcal{T}\left(\vec{\omega}_{d}\right)$. In most cases we must estimate $\mathcal{T}(\vec{\omega})$ or $\mathcal{T}\left(\vec{\omega}_{d}\right)$ numerically by calculating $T(\xi, \eta, \theta, \vec{\omega})$ over a discrete set of $\xi, \eta$, $\theta$ values in the cube $-\pi \leq \xi, \eta, \theta \leq \pi$ and take the maximum over these values. This was done for two-dimensional analysis in [25].

For a system of equations, when $T$ is a matrix, the method is stable at some $\vec{\omega}$ if $T$ is diagonalizable and the spectral radius $r(T)$ is no larger than 1 . For the class of wave propagation schemes considered, the amplification matrix is not proved to be diagonalizable. For those problems we have considered, numerical studies indicate that $T$ has this property in major parts of the wave number domain. For those wave numbers where the matrix is indicated to be non-diagonalizable, $\left\|T^{n}\right\|$ is computed for different values of $n$. In these cases, $\left\|T^{n}\right\|$ stays nicely bounded, and the spectral radius is always less than unity. Based on these observations, the wave propagation schemes is said to be stable for given $\vec{\omega}$ if

$$
\mathcal{T}(\vec{\omega}) \equiv \max _{\xi, \eta, \theta} r(T(\xi, \eta, \theta, \vec{\omega}))=1
$$

where maximum is taken for $-\pi \leq \xi, \eta, \theta \leq \pi$. In general, this is only a necessary condition for stability in the system case, but we have not observed that it fails for our applications.

\subsection{Scalar advection}

We start with the advection equation (4), where $u, v$, and $w$ are positive constants. The directional Courant numbers are then

$$
\omega_{1}=u \frac{\Delta t}{\Delta x}, \quad \omega_{2}=v \frac{\Delta t}{\Delta y}, \quad \omega_{3}=w \frac{\Delta t}{\Delta z} .
$$




\begin{tabular}{|c||c|c|c|c|c|c|c|c|c|}
\hline \multicolumn{1}{|c||}{} & \multicolumn{9}{c|}{$\max _{\xi, \eta, \theta}|T|$ for } \\
$\omega_{d}$ & \multicolumn{10}{c|}{} \\
& $T^{100}$ & $T^{110}$ & $T^{111}$ & $T^{210}$ & $T^{211}$ & $T^{220}$ & $T^{221}$ & $T^{222}$ & LW \\
\hline 0.1 & 1.00 & 1.00 & 1.00 & 1.06 & 1.05 & 1.00 & 1.00 & 1.00 & 1.00 \\
\hline 0.2 & 1.00 & 1.00 & 1.00 & 1.24 & 1.18 & 1.00 & 1.00 & 1.00 & 1.00 \\
\hline 0.3 & 1.00 & 1.00 & 1.00 & 1.54 & 1.32 & 1.00 & 1.00 & 1.00 & 1.03 \\
\hline 0.4 & 1.40 & 1.00 & 1.00 & 1.96 & 1.45 & 1.00 & 1.00 & 1.00 & 1.17 \\
\hline 0.5 & 2.00 & 1.00 & 1.00 & 2.50 & 1.50 & 1.00 & 1.50 & 1.00 & 1.57 \\
\hline 0.6 & 2.60 & 1.72 & 1.00 & 3.16 & 1.43 & 1.06 & 2.02 & 1.00 & 2.21 \\
\hline 0.7 & 3.20 & 2.68 & 1.00 & 3.94 & 1.26 & 1.37 & 2.33 & 1.00 & 3.01 \\
\hline 0.8 & 3.80 & 3.88 & 1.00 & 4.84 & 1.11 & 2.00 & 2.33 & 1.00 & 3.97 \\
\hline 0.9 & 4.40 & 5.32 & 1.00 & 5.86 & 1.02 & 3.92 & 1.92 & 1.00 & 5.08 \\
\hline 1.0 & 5.00 & 7.00 & 1.00 & 7.00 & 1.00 & 7.00 & 1.00 & 1.00 & 6.32 \\
\hline 1.01 & 5.06 & 7.18 & 1.06 & 7.12 & 1.12 & 7.37 & 1.14 & 1.12 & 6.45 \\
\hline
\end{tabular}

Table 3: The amplification factor $\mathcal{T}\left(\vec{\omega}_{d}\right)$ versus the CFL number $\omega_{d}=\omega_{1}=\omega_{2}=\omega_{3}$ for the scalar problem.

As an example of this stability analysis, consider the donor cell upwind method

$$
\begin{aligned}
\bar{Q}_{I J K} & =Q_{I J K}-\omega_{1}\left(Q_{I J K}-Q_{I-1, J K}\right)-\omega_{2}\left(Q_{I J K}-Q_{I, J-1, K}\right)-\omega_{3}\left(Q_{I J K}-Q_{I J, K-1}\right) \\
& =Q_{I J K}-\omega_{1} D_{x} Q_{I J K}-\omega_{2} D_{y} Q_{I J K}-\omega_{3} D_{z} Q_{I J K},
\end{aligned}
$$

where $D_{x}, D_{y}, D_{z}$ denote the upwind difference operators. This is the wave-propagation Method $(1,0,0)$ from Section 2. Inserting the Fourier mode (62) into this method yields $T(\xi, \eta, \theta, \vec{\omega})$ :

$$
T^{100}=1-\omega_{1}\left(1-e^{-i \xi}\right)-\omega_{2}\left(1-e^{-i \eta}\right)-\omega_{3}\left(1-e^{-i \theta}\right) .
$$

Here and below the superscripts on $T$ and $\mathcal{T}(\vec{\omega})$ refer to the labels $\left(m_{1}, m_{2}, m_{3}\right)$ denoting the method. This donor cell method can be shown to be stable only when

$$
\omega_{1}+\omega_{2}+\omega_{3} \leq 1
$$

which is more restrictive than our desired bound (61). In particular, along the diagonal we find that

$$
\mathcal{T}\left(\vec{\omega}_{d}\right)=\max \left(1,\left|1-6 \omega_{d}\right|\right) .
$$

so that the method is only stable for $\omega_{d} \leq 1 / 3$.

In Table 3 we present the values of $\mathcal{T}\left(\vec{\omega}_{d}\right)$ found numerically for each method of the type presented in Section 2, and also for the classical Lax-Wendroff method. The column labelled $T^{100}$, for example, shows that the method is stable for $\omega_{d}=0.3$ but not for $\omega_{d}=0.4$, since in the latter case the amplification factor is 1.40 .

We can improve the stability properties of the upwind algorithm by moving up to Method $(1,1,0)$, in which we introduce transverse propagation of the increment waves into adjacent cells. The amplification factor is then

$$
\begin{aligned}
T^{110} & =T^{100}+\omega_{1} \omega_{2}\left(1-e^{-i \xi}\right)\left(1-e^{-i \eta}\right)+\omega_{1} \omega_{3}\left(1-e^{-i \xi}\right)\left(1-e^{-i \theta}\right) \\
& +\omega_{2} \omega_{3}\left(1-e^{-i \eta}\right)\left(1-e^{-i \theta}\right) .
\end{aligned}
$$


In the diagonal case we find that the amplification factor is maximized by taking $\xi=\eta=\theta=\pi$ which results in

$$
\mathcal{T}^{110}\left(\vec{\omega}_{d}\right)=\max \left(1,\left|1-6 \omega_{d}+12 \omega_{d}^{2}\right|\right),
$$

and hence the method is stable for $\omega_{d} \leq 1 / 2$ as indicated in Table 3 .

By also introducing double-transverse propagation, as discussed in Section 2.3.1, we obtain Method $(1,1,1)$. It is possible to write $T^{111}$ in the compact form

$$
T^{111}=\left(1-\omega_{1}\left(1-e^{-i \xi}\right)\right)\left(1-\omega_{2}\left(1-e^{-i \eta}\right)\right)\left(1-\omega_{3}\left(1-e^{-i \theta}\right)\right) .
$$

It can easily be shown that $\mathcal{T}^{111}(\vec{\omega})=1$ for all $\vec{\omega}$ in the cube (61) and hence this method has optimal stability. (This is expected since Method $(1,1,1)$ is the "shift and average" scheme.)

Note that even though Method $(1,1,1)$ is only first order accurate, it includes approximations to the third derivative term $q_{x y z}$ coming from the double-transverse propagation (and apparent from the product $\omega_{1} \omega_{2} \omega_{3}$ appearing in the expression for $T^{111}$ ). We will see for second order methods that obtaining optimal stability requires including approximations to certain fourth order derivatives (obtained by the double-transverse propagation of correction waves in Method $(2,2,2))$.

\subsection{Second-order methods}

The simplest wave-propagation method which is second order accurate is Method $(2,1,0)$. Taking $m_{1}=2$ includes correction waves modeling the second derivative terms $q_{x x}, q_{y y}$ and $q_{z z}$ in the Taylor expansion. Taking $m_{2}=1$ gives transverse propagation of the increment waves, which models the cross-derivative terms $q_{x y}, q_{y z}$, and $q_{x z}$. The amplification matrix is

$$
\begin{aligned}
T^{210} & =T^{110}+\frac{1}{2} \omega_{1}\left(1-\omega_{1}\right)\left(1-e^{-i \xi}\right)\left(1-e^{i \xi}\right)+\frac{1}{2} \omega_{2}\left(1-\omega_{2}\right)\left(1-e^{-i \eta}\right)\left(1-e^{i \eta}\right) \\
& +\frac{1}{2} \omega_{3}\left(1-\omega_{3}\right)\left(1-e^{-i \theta}\right)\left(1-e^{i \theta}\right) .
\end{aligned}
$$

In the diagonal case we find

$$
T^{210}\left(\pi, \pi, \pi, \vec{\omega}_{d}\right)=1+6 \omega_{d}^{2}
$$

and hence this method is unstable for any $\omega_{d}$. The method is stable in the special case where one of the velocities is zero, say $w=0$ (and hence the corresponding directional Courant number is also zero, $\omega_{3}=0$ ). In this case the problem reduces to a two-dimensional problem and this method is optimally stable in two dimensions (this is what was called Method 3 in $[25])$. So Method $(2,1,0)$ is stable on faces of the cube $(61)$, e.g., for all $\vec{\omega}=\left(\omega_{1}, \omega_{2}, 0\right)$ with $0 \leq \omega_{1}, \omega_{2} \leq 1$, but nowhere in the interior as indicated in Table 3 and by the diagonal. Note that (68) gives the values in the table. This indicates that for this scheme the maximum is taken for wave numbers equal $\pi$.

In a fully three-dimensional problem our experience with first-order methods indicates that good stability requires including the corner-coupling terms $q_{x y z}$, and hence we must at least take $m_{3}=1$. The next simplest method includes transverse and double-transverse propagation of the increment waves, along with correction waves in the normal direction, and is Method $(2,1,1)$. The expression for $T^{211}$ is given by $(67)$ with $T^{110}$ replaced by $T^{111}$. Unfortunately this scheme is still unconditionally unstable for a fully three-dimensional problem, although the amplification factors are smaller than for Method $(2,1,0)$. From the table also note that 
the scheme has an isolated point of stability at $\omega_{d}=1.0$. For this value the scheme happens to be exact.

Transverse propagation of the correction wave must also be used in order to obtain a stable second order accurate method for fully three-dimensional problems. This means that $m_{2}=2$ and perhaps also $m_{3}=2$.

Method $(2,2,0)$ has amplification factor

$$
\begin{aligned}
T^{220} & =T^{210}+\frac{1}{2} \omega_{1} \omega_{2}\left(1-\omega_{1}\right)\left(e^{i \xi}-1\right)\left(1-e^{-i \xi}\right)\left(1-e^{-i \eta}\right) \\
& +\frac{1}{2} \omega_{1} \omega_{3}\left(1-\omega_{1}\right)\left(e^{i \xi}-1\right)\left(1-e^{-i \xi}\right)\left(1-e^{-i \theta}\right) \\
& +\frac{1}{2} \omega_{1} \omega_{2}\left(1-\omega_{2}\right)\left(e^{i \eta}-1\right)\left(1-e^{-i \eta}\right)\left(1-e^{-i \xi}\right) \\
& +\frac{1}{2} \omega_{2} \omega_{3}\left(1-\omega_{2}\right)\left(e^{i \eta}-1\right)\left(1-e^{-i \eta}\right)\left(1-e^{-i \theta}\right) \\
& +\frac{1}{2} \omega_{1} \omega_{3}\left(1-\omega_{3}\right)\left(e^{i \theta}-1\right)\left(1-e^{-i \theta}\right)\left(1-e^{-i \xi}\right) \\
& +\frac{1}{2} \omega_{2} \omega_{3}\left(1-\omega_{3}\right)\left(e^{i \theta}-1\right)\left(1-e^{-i \theta}\right)\left(1-e^{-i \eta}\right) .
\end{aligned}
$$

According to Table 3, this scheme is stable for $\omega_{d} \leq 0.5$, which is reasonable but still not optimal.

In Method $(2,2,1)$, the increment wave propagates as in Method $(1,1,1)$. The amplification factor is given by (69) but with $T^{210}$ replaced by $T^{211}$. Like Method $(2,1,0), T^{211}$ takes its maximum in the unstable regime for wave numbers equal $\pi$. In this case $T^{221}\left(\pi, \pi, \pi, \vec{\omega}_{d}\right)=$ $1-18 \omega_{d}^{2}+16_{d} \omega^{3}$ and the method is stable for $\omega_{d} \leq(1+\sqrt{33}) / 16 \approx 0.4215$, in agreement with the table. Compared to Method $(2,2,0)$, the stability region is slightly reduced, but the amplification factor outside of the stable region is smaller and, as for Method $(2,1,1)$, the scheme is exact for $\omega_{d}=1.0$.

Finally, if the correction wave also propagates in a three-dimensional manner we obtain the full Method $(2,2,2)$. The amplification factor for this method is

$$
\begin{aligned}
T^{222} & =T^{221}-\frac{1}{2} \omega_{1} \omega_{2} \omega_{3}\left(1-\omega_{1}\right)\left(e^{i \xi}-1\right)\left(1-e^{-i \xi}\right)\left(1-e^{-i \eta}\right)\left(1-e^{-i \theta}\right) \\
& -\frac{1}{2} \omega_{1} \omega_{2} \omega_{3}\left(1-\omega_{2}\right)\left(e^{i \eta}-1\right)\left(1-e^{-i \eta}\right)\left(1-e^{-i \xi}\right)\left(1-e^{-i \theta}\right) \\
& -\frac{1}{2} \omega_{1} \omega_{2} \omega_{3}\left(1-\omega_{3}\right)\left(e^{i \theta}-1\right)\left(1-e^{-i \theta}\right)\left(1-e^{-i \xi}\right)\left(1-e^{-i \eta}\right) .
\end{aligned}
$$

According to Table 3, the wave propagation scheme is stable for $\omega_{d} \leq 1$, which is the best one can expect.

\subsection{Other second order discretizations}

In this section we compare the stability results found for the wave-propagation algorithms with that of the standard Lax-Wendroff method. We also consider what happens if the centered approximations to second derivatives used in Lax-Wendroff, which forms the basis for the correction waves in our methods, are replaced by other standard approximations such as those used in the 1-dimensional Beam-Warming or Fromm methods. 
Consider a general method of the form

$$
\bar{Q}_{i j k}=Q_{i j k}-\omega_{1} D_{x} Q_{i j k}-\omega_{2} D_{y} Q_{i j k}-\omega_{3} D_{z} Q_{i j k}+E_{1}+E_{2}
$$

The term $E_{1}$ includes the approximations of the pure second order derivatives in the Taylor series while $E_{2}$ models cross-derivative terms. Taking $E_{1}=E_{2}=0$ gives the Donor Cell upwind method, while the standard Lax-Wendroff method has

$$
\begin{aligned}
E_{1}^{L W}= & -\frac{1}{2} \omega_{1}\left(1-\omega_{1}\right)\left(D_{x} Q_{i+1, j k}-D_{x} Q_{i j k}\right)-\frac{1}{2} \omega_{2}\left(1-\omega_{2}\right)\left(D_{y} Q_{i, j+1, k}-D_{y} Q_{i j k}\right) \\
& -\frac{1}{2} \omega_{3}\left(1-\omega_{3}\right)\left(D_{z} Q_{i j k+1}-D_{z} Q_{i j k}\right) . \\
E_{2}^{L W}= & \omega_{1} \omega_{2} D_{y}^{c} D_{x}^{c} Q_{i j k}+\omega_{1} \omega_{3} D_{z}^{c} D_{x}^{c} Q_{i j k}+\omega_{2} \omega_{3} D_{z}^{c} D_{y}^{c} Q_{i j k}
\end{aligned}
$$

where $D_{x}^{c} Q_{i j k}$ denotes the centered difference $\left(Q_{i+1, j k}-Q_{i-1, j k}\right) / 2$. The Lax-Wendroff amplification factor is

$$
\begin{aligned}
T^{L W} & =1-\frac{1}{2} \omega_{1}\left(e^{i \xi}-e^{-i \xi}\right)-\frac{1}{2} \omega_{2}\left(e^{i \eta}-e^{-i \eta}\right)-\frac{1}{2} \omega_{3}\left(e^{i \omega}-e^{-i \omega}\right) \\
& +\frac{1}{2} \omega_{1}^{2}\left(e^{i \xi}-1\right)\left(1-e^{-i \xi}\right)+\frac{1}{2} \omega_{2}^{2}\left(e^{i \eta}-1\right)\left(1-e^{-i \eta}\right)+\frac{1}{2} \omega_{3}^{2}\left(e^{i \omega}-1\right)\left(1-e^{-i \omega}\right) \\
& +\frac{1}{4} \omega_{1} \omega_{2}\left(e^{i \xi}-e^{-i \xi}\right)\left(e^{i \eta}-e^{-i \eta}\right)+\frac{1}{4} \omega_{1} \omega_{3}\left(e^{i \xi}-e^{-i \xi}\right)\left(e^{i \omega}-e^{-i \omega}\right) \\
& +\frac{1}{4} \omega_{2} \omega_{3}\left(e^{i \eta}-e^{-i \eta}\right)\left(e^{i \omega}-e^{-i \omega}\right) .
\end{aligned}
$$

Along the diagonal, Lax-Wendroff is stable for $\omega_{d} \leq 0.1925$.

One might attempt to improve stability by replacing the centered approximations to crossderivative terms by upwind approximations, using

$$
E_{2}^{u p}=\omega_{1} \omega_{2} D_{y} D_{x} Q_{i j k}+\omega_{1} \omega_{3} D_{z} D_{x} Q_{i j k}+\omega_{2} \omega_{3} D_{z} D_{y} Q_{i j k}
$$

in place of $E_{2}^{L W}$. This gives Method $(2,1,0)$ which was seen above to be unconditionally unstable. However, if we also replace $E_{1}^{L W}$ by an upwind-biased approximation, stability can be restored. One possibility is to use the fully-upwind Beam-Warming approximation

$$
\begin{aligned}
E_{1}^{B W}= & -\frac{1}{2} \omega_{1}\left(1-\omega_{1}\right)\left(D_{x} Q_{i j k}-D_{x} Q_{i-1, j k}\right)-\frac{1}{2} \omega_{2}\left(1-\omega_{2}\right)\left(D_{y} Q_{i j k}-D_{y} Q_{i, j-1, k}\right) \\
& -\frac{1}{2} \omega_{3}\left(1-\omega_{3}\right)\left(D_{z} Q_{i j k}-D_{z} Q_{i j, k-1}\right) .
\end{aligned}
$$

while another is to use centered differences such as $D_{x}^{c} Q_{I J K}$ in place of the upwind difference $D_{x} Q_{I J K}$ in $E_{1}^{L W}$. This corresponds to Fromm's method in one dimension, so we call this

$$
\begin{aligned}
E_{1}^{F r}= & -\frac{1}{4} \omega_{1}\left(1-\omega_{1}\right)\left(D_{x}^{c} Q_{i j k}-D_{x}^{c} Q_{i-1, j k}\right)-\frac{1}{4} \omega_{2}\left(1-\omega_{2}\right)\left(D_{y}^{c} Q_{i j k}-D_{y}^{c} Q_{i, j-1, k}\right) \\
& -\frac{1}{4} \omega_{3}\left(1-\omega_{3}\right)\left(D_{z}^{c} Q_{i j}-D_{z}^{c} Q_{i j, k-1}\right) .
\end{aligned}
$$

Replacing $E_{1}^{L W}$ by one of these and using $E_{2}^{u p}$ gives methods we label Method BW(2,1,0) and Method $\operatorname{Fr}(2,1,0)$ for Beam-Warming and Fromm, respectively. One could then add in 


\begin{tabular}{|c||c|c|c|c|c|}
\hline \multicolumn{1}{|c||}{} & \multicolumn{5}{c|}{$\max _{\xi, \eta, \theta}|T|$, Beam-Warming } \\
$\omega_{d}$ & \multicolumn{1}{c|}{$T^{210}$} & $T^{211}$ & $T^{220}$ & $T^{221}$ & $T^{222}$ \\
\hline 0.1 & 1.00 & 1.00 & 1.00 & 1.00 & 1.00 \\
\hline 0.2 & 1.00 & 1.00 & 1.00 & 1.00 & 1.00 \\
\hline 0.3 & 1.00 & 1.20 & 1.00 & 1.00 & 1.00 \\
\hline 0.4 & 1.00 & 1.43 & 1.38 & 1.00 & 1.00 \\
\hline 0.5 & 1.00 & 1.50 & 2.50 & 1.50 & 1.00 \\
\hline 0.6 & 1.00 & 1.45 & 3.74 & 2.01 & 1.00 \\
\hline 0.7 & 1.42 & 1.32 & 4.95 & 2.20 & 1.00 \\
\hline 0.8 & 2.92 & 1.18 & 5.99 & 1.90 & 1.00 \\
\hline 0.9 & 4.78 & 1.05 & 6.72 & 1.19 & 1.00 \\
\hline 1.0 & 7.00 & 1.00 & 7.00 & 1.00 & 1.00 \\
\hline 1.01 & 7.24 & 1.08 & 7.00 & 1.25 & 1.00 \\
\hline
\end{tabular}

\begin{tabular}{|c||c|c|c|c|c|}
\hline \multicolumn{1}{|c||}{} & \multicolumn{5}{c|}{$\max _{\xi, \eta, \theta}|T|$, Fromm } \\
$\omega_{d}$ & \multicolumn{1}{c||}{$T^{210}$} & $T^{211}$ & $T^{220}$ & $T^{221}$ & $T^{222}$ \\
\hline 0.1 & 1.00 & 1.00 & 1.00 & 1.00 & 1.00 \\
\hline 0.2 & 1.00 & 1.00 & 1.00 & 1.00 & 1.00 \\
\hline 0.3 & 1.00 & 1.00 & 1.00 & 1.00 & 1.00 \\
\hline 0.4 & 1.00 & 1.00 & 1.00 & 1.00 & 1.00 \\
\hline 0.5 & 1.00 & 1.00 & 1.00 & 1.00 & 1.00 \\
\hline 0.6 & 1.72 & 1.00 & 1.72 & 1.01 & 1.00 \\
\hline 0.7 & 2.68 & 1.00 & 2.68 & 1.03 & 1.00 \\
\hline 0.8 & 3.88 & 1.00 & 3.88 & 1.02 & 1.00 \\
\hline 0.9 & 5.32 & 1.00 & 5.32 & 1.02 & 1.00 \\
\hline 1.0 & 7.00 & 1.00 & 7.00 & 1.00 & 1.00 \\
\hline 1.01 & 7.18 & 1.06 & 7.18 & 1.11 & 1.06 \\
\hline
\end{tabular}

Table 4: The amplification factor $\mathcal{T}\left(\vec{\omega}_{d}\right)$ versus the CFL number $\omega_{d}=\omega_{1}=\omega_{2}=\omega_{3}$ for the scalar problem. In the left table, Beam-Warming type discretization is used for (pure) second order derivatives. In the left table, Fromm discretization is used.

transverse propagation of the correction terms and perhaps double-transverse propagation as well, giving methods such as BW $(2,2,2)$, etc. Only minor changes have to be made in the expressions for the amplification factors $T^{210}, T^{211}, T^{220}, T^{221}$, and $T^{222}$, in order to account for this change in the underlying approximation. For the BW-type approximation, the change is

$$
\text { replace }\left(e^{i \xi}-1\right)\left(1-e^{-i \xi}\right) \text { by }\left(e^{i \xi}-1\right)\left(1-e^{-i \xi}\right) e^{-i \xi} \text {. }
$$

Similar changes have to be made for corresponding terms involving $\eta$ and $\theta$. Likewise, the changes to be made for the inclusion of Fromm-type discretization are

$$
\text { replace }\left(e^{i \xi}-1\right)\left(1-e^{-i \xi}\right) \quad \text { by } \quad \frac{1}{2}\left(1-e^{-i \xi}\right)\left(e^{i \xi}-e^{-i \xi}\right) .
$$

These modified methods are interesting to study since the effect of adding certain limiters is to give an upwind bias towards one of these methods. The full effect of adding a limiter is more complicated since the methods are then nonlinear and von Neumann analysis does not apply, but nonetheless it may be reassuring to know that we are switching to a method which is also stable in its own right.

Table 4 gives a summary of stability results along the diagonal for these modified methods. One significant result is that none of these methods is unconditionally unstable in the manner of Method LW $(2,1,0)$ and LW $(2,1,1)$. In general the Fromm methods have the largest stability regions, though in the case of Method $(2,2,2)$ all three approaches give optimally stable methods.

\subsection{Linearized Euler equations (acoustics)}

In the previous sections we have studied stability for the scalar advection equation. Applying the wave-propagation algorithms to a system of equations is considerably more complicated 
since solving each Riemann problem now corresponds to splitting jumps into eigenvectors. For a particular linear system it is still possible to analyze stability using the von Neumann approach by computing the amplification matrices which arise. As an example, we consider a linearization of the Euler equations in the primitive variables $\rho, u, v, w$, and $p$. The equations are linearized using the constant state $\left(\rho_{0}, u_{0}, v_{0}, w_{0}, p_{0}\right)$. This gives the equations for acoustics in a uniformly-moving flow. The matrices involved are

$$
\begin{gathered}
A=\left[\begin{array}{ccccc}
u_{0} & \rho_{0} & 0 & 0 & 0 \\
0 & u_{0} & 0 & 0 & 1 / \rho_{0} \\
0 & 0 & u_{0} & 0 & 0 \\
0 & 0 & 0 & u_{0} & 0 \\
0 & \gamma p_{0} & 0 & 0 & u_{0}
\end{array}\right], \quad B=\left[\begin{array}{ccccc}
v_{0} & 0 & \rho_{0} & 0 & 0 \\
0 & v_{0} & 0 & 0 & 0 \\
0 & 0 & v_{0} & 0 & 1 / \rho_{0} \\
0 & 0 & 0 & v_{0} & 0 \\
0 & 0 & \gamma p_{0} & 0 & u_{0}
\end{array}\right] \\
C=\left[\begin{array}{ccccc}
w_{0} & 0 & 0 & \rho_{0} & 0 \\
0 & w_{0} & 0 & 0 & 0 \\
0 & 0 & w_{0} & 0 & 0 \\
0 & 0 & 0 & w_{0} & 1 / \rho_{0} \\
0 & 0 & 0 & \gamma p_{0} & w_{0}
\end{array}\right]
\end{gathered}
$$

The eigenvalues are $\lambda=\left\{u_{0}-c_{0}, u_{0}, u_{0}, u_{0}, u_{0}+c_{0}\right\}, \mu=\left\{v_{0}-c_{0}, v_{0}, v_{0}, v_{0}, v_{0}+c_{0}\right\}$, and $\nu=\left\{w_{0}-c_{0}, w_{0}, w_{0}, w_{0}, w_{0}+c_{0}\right\}$. Here $c_{0}=\sqrt{\gamma p_{0} / \rho_{0}}$ denotes the sound speed.

The stability region depends on these constant values. Taking $u_{0}=v_{0}=w_{0}=0, \gamma p_{0}=$ $\rho_{0}=1$, the stability results turn out to be very close to what was achieved for the scalar problem, in spite of the fact that this is a fully coupled system of acoustics equations.

Here we present a slightly more interesting case in which the background velocity is nonzero, so acoustic waves move relative to a fixed flow field. The following values are used: $u_{0}=v_{0}=$ $w_{0}=1, \gamma p_{0}=2$ and $\rho_{0}=1$.

The expressions for $T(\xi, \eta, \theta, \vec{\omega})$ are not hard to derive, but involve a large number of terms as the algorithm includes an increasing number of updates of neighboring cells. For example, the amplification matrix for the very simple Method $(1,0,0)$ reads

$$
\begin{aligned}
T^{100} & =I-\frac{\Delta t}{\Delta x}\left[A^{+}\left(1-e^{-i \xi}\right)+A^{-}\left(e^{i \xi}-1\right)\right] \\
& -\frac{\Delta t}{\Delta y}\left[B^{+}\left(1-e^{-i \eta}\right)+B^{-}\left(e^{i \eta}-1\right)\right] \\
& -\frac{\Delta t}{\Delta z}\left[C^{+}\left(1-e^{-i \theta}\right)+C^{-}\left(e^{i \theta}-1\right)\right] .
\end{aligned}
$$

The spectral radius of the amplification matrix for each method on this linear system is given in Table 5. With the constant state used in the linearization, the Courant number $\omega_{d}$ is given by

$$
\omega_{d}=(1+\sqrt{2}) \frac{\Delta t}{\Delta x},
$$

assuming that equal spacing is used in all directions, i.e. $\Delta x=\Delta y=\Delta z$.

The results are roughly the same as for scalar advection. Transverse propagation of the increment waves in Method (1,1,0) adds stability compared to the simple Method (1,0,0). Adding double-transverse propagation of the increment waves gives the optimally stable Method $(1,1,1)$. 


\begin{tabular}{|c||c|c|c|c|c|c|c|c|c|}
\hline \multicolumn{1}{|c||}{} & \multicolumn{9}{c|}{$\max _{\xi, \eta, \theta}(r(T))$ for } \\
$\omega_{d}$ & \multicolumn{10}{c|}{$T^{100}$} & $T^{110}$ & $T^{111}$ & $T^{210}$ & $T^{211}$ & $T^{220}$ & $T^{221}$ & $T^{222}$ & LW \\
\hline 0.1 & 1.00 & 1.00 & 1.00 & 1.03 & 1.02 & 1.00 & 1.00 & 1.00 & 1.00 \\
\hline 0.2 & 1.00 & 1.00 & 1.00 & 1.10 & 1.08 & 1.00 & 1.00 & 1.00 & 1.00 \\
\hline 0.3 & 1.00 & 1.00 & 1.00 & 1.23 & 1.15 & 1.00 & 1.00 & 1.00 & 1.00 \\
\hline 0.4 & 1.00 & 1.00 & 1.00 & 1.41 & 1.22 & 1.00 & 1.00 & 1.00 & 1.02 \\
\hline 0.5 & 1.32 & 1.00 & 1.00 & 1.63 & 1.28 & 1.00 & 1.00 & 1.00 & 1.12 \\
\hline 0.6 & 1.79 & 1.00 & 1.00 & 1.91 & 1.31 & 1.00 & 1.05 & 1.00 & 1.37 \\
\hline 0.7 & 2.25 & 1.01 & 1.00 & 2.24 & 1.31 & 1.00 & 1.37 & 1.00 & 1.80 \\
\hline 0.8 & 2.72 & 1.53 & 1.00 & 2.62 & 1.37 & 1.01 & 1.55 & 1.00 & 2.34 \\
\hline 0.9 & 3.18 & 2.19 & 1.00 & 3.05 & 1.42 & 1.17 & 1.53 & 1.00 & 2.99 \\
\hline 1.0 & 3.65 & 2.98 & 1.00 & 3.54 & 1.46 & 1.63 & 1.23 & 1.00 & 3.74 \\
\hline 1.01 & 3.69 & 3.07 & 1.02 & 3.59 & 1.47 & 1.72 & 1.23 & 1.04 & 3.82 \\
\hline
\end{tabular}

Table 5: The amplification factor $\mathcal{T}\left(\vec{\omega}_{d}\right)$ versus the CFL number $\omega_{d}$ for the linearized Euler system.

Replacing the centered approximations of cross-derivatives in the Lax-Wendroff scheme with upwind approximations results in an unconditionally unstable Method $(2,1,0)$. The degree of instability is reduced in Method $(2,1,1)$, but still the scheme is unconditionally unstable. Introducing transverse propagation of the correction wave adds stability. Still, the stability area of Method $(2,2,0)$ is larger than for Method $(2,2,1)$, but the latter has less pronounced instability than was observed for the scalar example. When both the increment wave and the correction wave propagate in a three-dimensional manner we obtain Method $(2,2,2)$, a method which is again optimally stable.

Note that the difference in stability between Method $(2,2,0)$ and Method $(2,2,2)$ is less than for the scalar problem. From experiments involving other parameters and equations, the most restrictive stability limit for Method $(2,2,0)$ appears to be $\omega_{d} \leq 1 / 2$. As discussed in Section 2, the updates resulting from one single interface in Method $(2,2,2)$ involve 15 Riemann problems, while the same number for Method $(2,2,0)$ is 5 . Hence, Method $(2,2,0)$ may, for certain applications, be computationally more efficient than Method $(2,2,2)$.

Recall from the previous sections that the transverse propagation of the correction wave gives rise to an approximation of several terms from a Taylor expansion added together. This is obtained under the assumption that the matrices involved commute. The matrices in the system (74) do not commute, but the stability does not seem to suffer from this. This is also experienced when considering other systems. At least for these cases, the stability limit appears to be insensitive to the commutation of the matrices.

\subsection{Limiter influence and wave number dependency}

The numerical example in Section 3.2 was also studied in [18]. The method used was $(2,1,1)$ at a Courant number of about 0.9 , and the computation did not suffer from any instability problems. From other numerical experiments we have observed that the unconditionally unstable methods $(2,1,0)$ and $(2,1,1)$ do not behave as badly as the discussion above may indicate. 

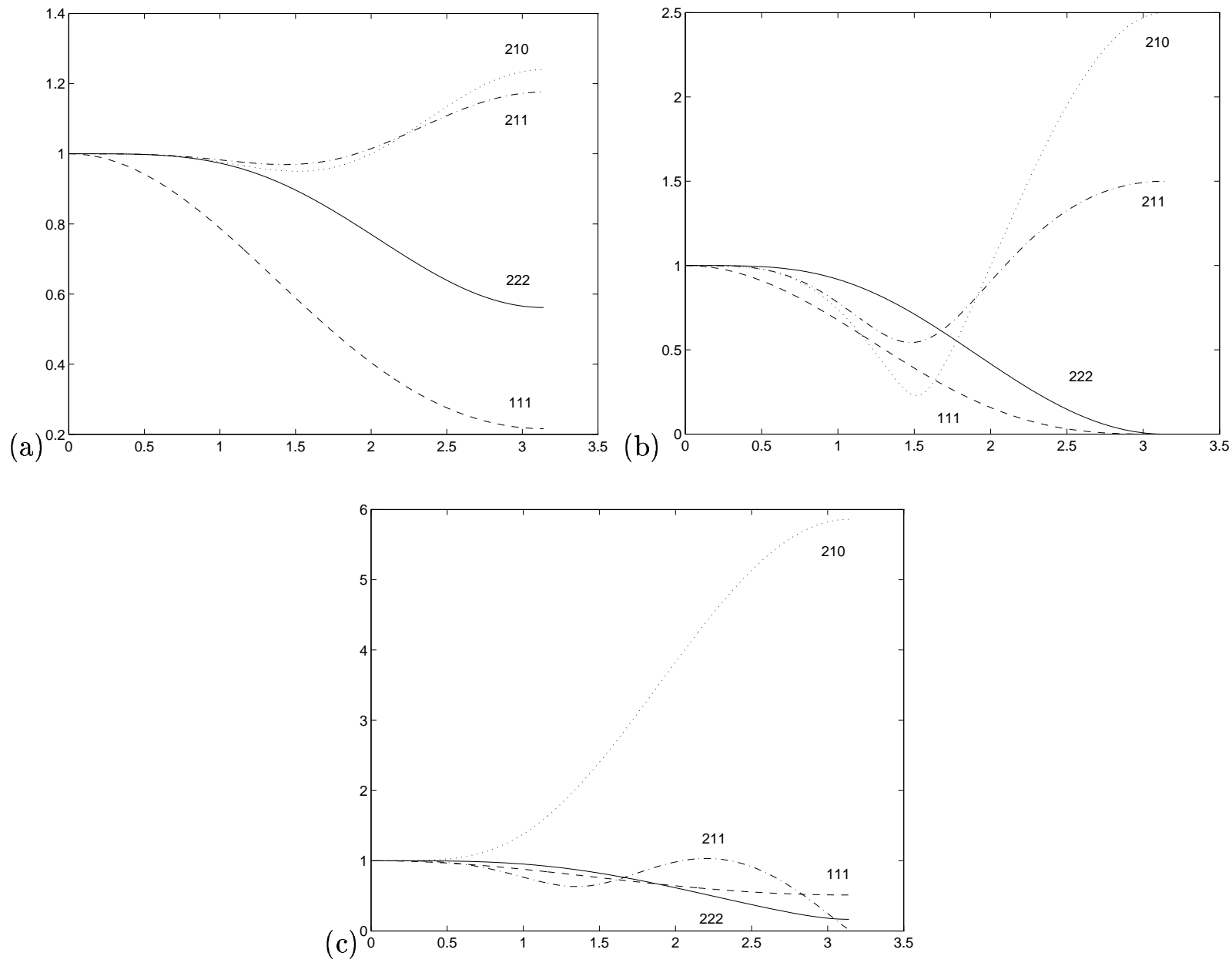

Figure 10: The absolute value of the amplification factor versus the wave number $\xi=\eta=\theta$ for the scalar problem. The Courant numbers are held fixed: (a) $\omega_{d}=0.2$, (b) $\omega_{d}=0.5$, (c) $\omega_{d}=0.9$.

This is especially true for Method $(2,1,1)$. But even Method $(2,1,0)$ may produce nice results when applied on nonlinear problems if limiters are used. This indicates that the instability is strongest for large wave numbers. Limiters are assumed to be most effective in this range. In this section we reconsider the two test problems from the previous section, and examine how the amplification factor depends on wave numbers and to some extent on limiters.

In order to simplify the discussion, we consider the case when the wave numbers are equal, i.e. $\xi=\eta=\theta$. In Figure 10, the absolute value of the amplification factor versus the wave number for the scalar problem is depicted for three different values of the Courant number $\omega_{d}$. The analytical expressions derived in the previous section are used. For all schemes, $|T|$ is symmetric about $\xi=0$, and in the figures only $0 \leq \xi \leq \pi$ is given.

As expected, the stable methods $(1,1,1)$ and $(2,2,2)$ are uniformly bounded by one, and the former scheme is more dissipative than the latter. When it comes to the unstable schemes, Method $(2,1,0)$ is more unstable than Method $(2,1,1)$ for nearly all wave numbers and Courant 
numbers.

These results may be utilized to find initial conditions that trigger instabilities in the schemes. Consider the initial condition

$$
q_{0}(x, y, z)=\sin (2 \pi n x+2 \pi n y+2 \pi n z)
$$

where $n$ is a positive integer. Assume that the computational domain is $[0,1] \times[0,1] \times[0,1]$, that $\Delta x=\Delta y=\Delta z$, and that periodic boundary conditions are used on all boundaries. The wavelength in the Fourier mode (62) is $2 \pi \Delta x / \xi$, and in (75) the wavelength is $1 / n$. Hence

$$
\xi=\frac{2 \pi n}{N},
$$

where $N$ is the number of cells in each coordinate direction. Since the shortest wavelength obtainable on the grid is $2 \Delta x$, acceptable values for $n$ are $1,2, \ldots, N / 2$, assuming that $N$ is even.

An estimate for the amplification factor is obtain by considering the ratio

$$
\max _{I J K}\left|\bar{Q}_{I J K}\right| / \max _{I J K}\left|Q_{I J K}\right| .
$$

An average value of this ratio for a number of steps is used. Due to roundoff errors, a computation may trigger instabilities, even though the wavenumbers used should give stability. In such cases, the average is estimated based on "stable steps".

In Figure 11, the estimated amplification factor is depicted with "o" symbols for Method $(2,1,1)$. In these computations $N=40$ and $n=5,10,15,20$ are used. The symbols lie very close to the curves as expected.

It is now possible to study the effect of limiters on stability. The computations are redone, but now with the MC-limiter turned on. The estimates for the ratio (76) are marked using $\mathrm{x}$-marks. The overall picture is that the limiters may prevent the solution from going unstable for all Courant numbers. Note that when $n=20$, yielding a wavelength of $2 \Delta x$, the gradient in the numerical solution changes sign from one cell to the other. In this case the limiter becomes zero, the high order terms are switched off and the scheme is equivalent to Method $(1,1,1)$.

In the previous section we also considered the linearized Euler equations. In Figure 12, the wave number dependency is depicted for some of the schemes for three different Courant numbers. Compared to the behavior of the schemes when applied to the scalar problem, the situation is clearer. For a given wave number, the tendency is that $r\left(T^{111}\right)<r\left(T^{222}\right)<$ $r\left(T^{211}\right)<r\left(T^{210}\right)$. Also note that for both the unstable schemes, $r(T)$ stays close to unity except for "large" wave numbers. This is especially the case for Method $(2,1,1)$. Since limiters tend to damp the influence of large wave numbers, the expected instability may be hard to observe when limiters are used.

Limiters are constructed in order to reduce oscillations near discontinuities. They are not intended to increase the stability range of the scheme. Nevertheless, it seems that the use of limiters has a positive effect on Method $(2,1,1)$. Similar computations as those reported above show that this also is the case to some extent for Method $(2,1,0)$. Note however that there is no reason to use Method $(2,1,1)$, since it involves nearly the same amount of work as the stable Method $(2,2,2)$. Method $(2,1,0)$ should probably not be trusted in general, even though it produces good looking results on certain problems. 

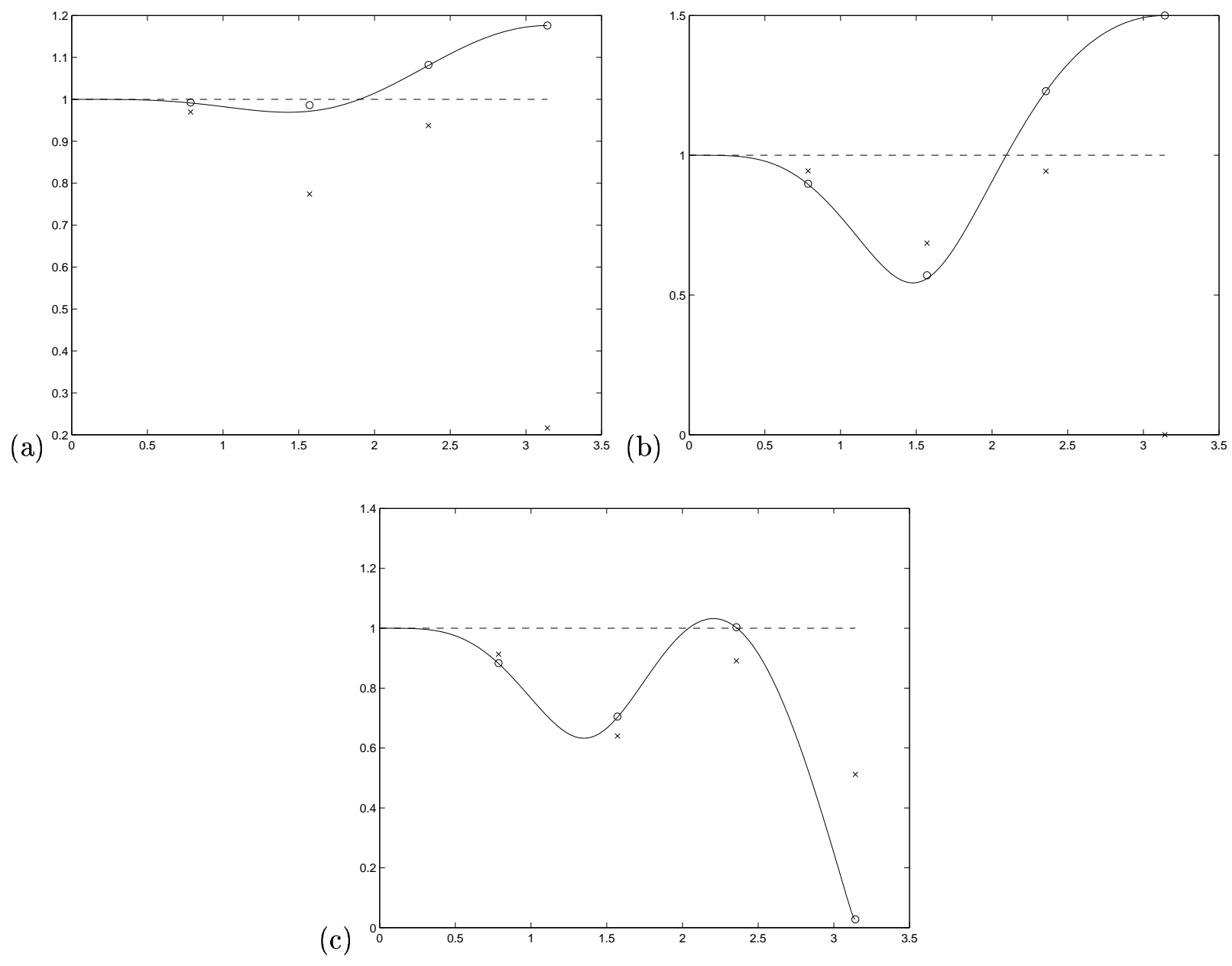

Figure 11: The absolute value of the amplification factor versus the wave number $\xi=\eta=\theta$ for Method $(2,1,1)$ applied to the scalar problem. The Courant numbers are: (a) $\omega_{d}=0.2$, (b) $\omega_{d}=0.5$, (c) $\omega_{d}=0.9$. The circles (o) correspond to numerical experiments, while the $\mathrm{x}$-marks show the same numerical experiments when the MC limiter is incorporated. 

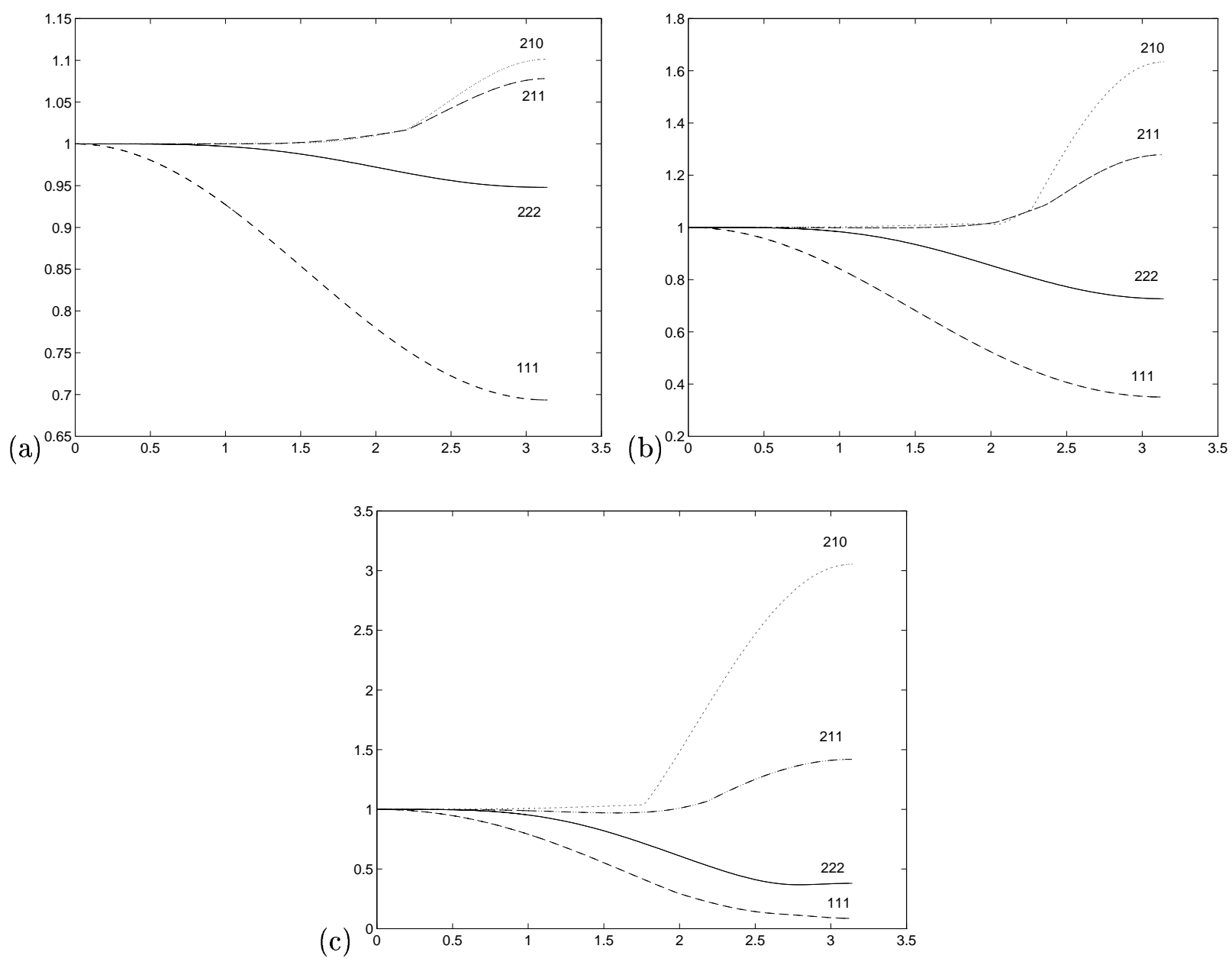

Figure 12: The spectral radius of the amplification matrix versus the wave number $\xi=\eta=\theta$ for the linearized Euler equations. The Courant numbers are held fixed: (a) $\omega_{d}=0.2$, (b) $\omega_{d}=0.5,\left(\right.$ c) $\omega_{d}=0.9$. 


\section{Conclusions}

In this paper, a class of three-dimensional wave propagation methods for conservation laws is constructed. By using proper wave propagation, a method is obtained which is stable for Courant number up to one. Simpler versions of the method appear to be stable in practice due to the effects of the nonlinear limiter function even though the unlimited versions are unstable for linear problems. This has been analyzed in Section 4.

The 3D scheme generalizes the two-dimensional approach for systems in [26], and the 3D scheme for scalar advection in [25]. The wave propagation method is implemented in Fortran, and is included in the software package CLAWPACK, freely available on the Web.

The methods have been applied to the Euler equations as a sample application. Though the examples used in this paper are written in conservative form, the methods, as implemented in CLAWPACK, handle the more general class of hyperbolic equations (3) as discussed in [26].

A three-dimensional version of the AMRCLAW package is also under development in joint work with Marsha Berger. This implements adaptive mesh refinement as described in two space dimensions in [4]. For large three-dimensional problems it may be necessary to use parallel processing. The ZPLCLAW package being developed with Turkiyyah and Wu [45] using the ZPL programming language of Snyder [39] may be useful in this context. Pointers to both AMRCLAW and ZPLCLAW can be found from the CLAWPACK webpage.

\section{References}

[1] T. J. Barth. On unstructured grids and solvers. Von Karman Institute for Fluid Dynamics Lecture Series, 90-03, 1990.

[2] J. B. Bell, C. N. Dawson, and G. R. Shubin, An unsplit, higher order Godunov method for scalar conservation laws in multiple dimensions, J. Comp. Phys., 74 (1988), pp. 1-24.

[3] M. J. Berger and R. J. LeVeque. AmRCLAW software. http://www . amath. washington. edu/ ${ }^{\sim} \mathrm{rj} 1 /$ amrclaw/.

[4] M. J. Berger and R. J. LeVeque. Adaptive mesh refinement using wave-propagation algorithms for hyperbolic systems. SIAM J. Numer. Anal., 35 (1998), pp. 2298-2316.

[5] S. J. Billett, A Class of Upwind Methods for Conservation Laws, Ph.D. Thesis, Cranfield University, College of Aeronautics, 1994.

[6] J. P. Boris, D. L. Book, Flux corrected transport I, SHASTA, a fluid transport algorithm that works, J. Comput. Phys., 11 (1973), pp. 38-69.

[7] P. Colella, Multidimensional upwind schemes for hyperbolic conservation laws, J. Comput. Phys., 87 (1990), pp. 171-200.

[8] P. Colella, P. Woodward, The piecewise-parabolic method (PPM) for gas-dynamical simulations, J. Comput. Phys., 54 (1984), pp. 174-201.

[9] M. Crandall and A. Majda, The method of fractional steps for conservation laws, Numer. Math. 34 (1980), 285-314. 
[10] Wai Sun Don, C. B. Quillen, Numerical simulation of shock-cylinder interactions: I. Resolution, J. Comp. Phys., 122 (1995), pp. 244-265.

[11] T. Fogarty, R. J. LeVeque, High-Resolution Finite Volume Methods for Acoustics in a Rapidly-Varying Heterogeneous Medium, Proc. Fourth Int. Conf. on Wave Propagation, Golden, CO, SIAM, 1998,

(ftp://amath.washington.edu/pub/rjl/papers/tf-rjl:wave98.ps.Z)

[12] T. Fogarty, R. J. LeVeque, High-resolution finite volume methods for acoustics in periodic or random media, to appear in J. Acoust. Soc. Am., 1999.

[13] E. Godlewski, P.-A. Raviart, Numerical Approximation of Hyperbolic Systems of Conservation Laws, Springer-Verlag, New York, 1996.

[14] S. K. Godunov, Finite difference methods for numerical computations of discontinuous solutions of equations of fluid dynamics, Mat. Sb., 47 (1959), pp. 271-306 (in Russian).

[15] J. J. Gottlieb, P. T. Groth, Assessment of Riemann solvers for unsteady one-dimensional inviscid flows of perfect gases J. Comput. Phys. 78 (1988) pp. 437-458.

[16] A. Harten, J. M. Hyman, Self-adjusting grid methods for one-dimensional hyperbolic conservation laws, J. Comput. Phys., 50 (1983), pp. 235-269.

[17] D. Kröner, Numerical Schemes for Conservation Laws, Wiley-Teubner series, 1997.

[18] J. O. Langseth, R. J. LeVeque, Three-dimensional Euler computations using CLAWPACK, in Conf. on Numer. Meth. for Euler and Navier-Stokes Eq., Montreal, P. Arminjon, ed., 1995, to appear.

(ftp://amath.washington.edu/pub/rjl/papers/jol-rjl:montreal95.ps.Z)

[19] J. O. Langseth, Wave Propagation Methods, Operator Splitting, and Front Tracking for Hyperbolic Problems, Ph.D. Thesis, University of Oslo, Department of Informatics, 1996.

[20] P. D. Lax, Hyperbolic systems of conservation laws, II, Comm. Pure Appl. Math., 10 (1957), pp. 537-566.

[21] P. D. Lax and B. Wendroff, Difference schemes for hyperbolic equations with high order of accuracy, Comm. Pure Appl. Math., 18 (1964), pp. 381-398.

[22] B. P. Leonard, M. K. MacVean, and A. P. Lock, Positivity-preserving numerical schemes for multidimensional advection. NASA Technical Memorandum 106055, ICOMP-93-05, 1993.

[23] R. J. LeVeque, Numerical Methods for Conservation Laws, Birkhäuser Verlag, (1990).

[24] R. J. LeVeque, Simplified multi-dimensional flux limiter methods, in Numerical Methods for Fluid Dynamics 4. M. J. Baines and K. W. Morton, eds., Oxford University Press, 1993, pp. 175-190.

[25] R. J. LeVeque, High-resolution conservative algorithms for advection in incompressible flow, SIAM J. Numer. Anal., 33 (1996), pp. 627-665. 
[26] R. J. LeVeque, Wave propagation algorithms for multi-dimensional hyperbolic systems, J. Comput. Phys., 131 (1997), pp. 327-353.

[27] R. J. LeVeque, CLAWPACK software. Available from netlib.att.com in netlib/pdes/claw, or on the Web at the URL http://amath. washington.edu/ ${ }^{\sim} \mathrm{rjl}$ / clawpack.html.

[28] J. J. Quirk, S. Karni, On the dynamics of a shock-bubble interaction, ICASE Report No. 94-75.

[29] Y. B. Radvogin, Quasi-monotonous multidimensional differential schemes with second order accuracy. Soviet Academy of Science Preprint (in Russian), 1991.

[30] R. D. Richtmyer, K. W. Morton, Difference Methods for Initial-value Problems, WileyInterscience, 1967.

[31] P. L. Roe, Approximate Riemann solvers, parameter vectors, and difference schemes, J. Comput. Phys., 43 (1981), pp. 357-372.

[32] P. L. Roe and D. Sidilkover, Optimum positive linear schemes for advection in two and three dimensions, J. Numer. Anal., 29 (1992), pp. 1542-1568.

[33] P. L. Roe, Sonic flux formulae, SIAM J. Sci. Stat. Comput., 13 (1992), pp. 611-630.

[34] J. Saltzman, Monotonic difference schemes for the linear advection equation in two and three dimensions. Los Alamos Report LA-UR-87-2479, 1987

[35] J. Saltzman, An unsplit 3-D upwind method for hyperbolic conservation laws, J. Comput. Phys., 115 (1994), pp. 153-168.

[36] C. W. Schulz-Rinne, J. P. Collins, H. M. Glaz, Numerical solution of the Riemann problem for two-dimensional gas dynamics. Research Report No. 92-02, Seminar für Angewandte Mathematik, ETH Zürich, 1992.

[37] P. K. Smolarkiewicz and W. W. Grabowski, The multidimensional positive definite advection transport algorithm: nonoscillatory option, J. Comput. Phys., 86 (1990), pp. 355-375.

[38] J. Smoller, Shock waves and Reaction-Diffusion Equations, Springer, 1983.

[39] L. Snyder. A programmer's guide to ZPL. MIT Press, 1999.

[40] G. Strang, On the construction and comparison of difference schemes, SIAM J. Num. Anal., 5 (1968), pp. 506-517.

[41] J. C. Strikwerda, Finite Difference Schemes and Partial Differential Equations, Wadsworth \& Brooks/Cole, 1989.

[42] P. K. Sweby, High resolution schemes using flux limiters for hyperbolic conservation laws, SIAM J. Num. Anal., 21 (1984), pp. 995-1011.

[43] E. F. Toro, Riemann Solvers and Numerical Methods for Fluid Dynamics: A Practical Introduction, Springer-Verlag, Berlin-Heidelberg, 1997. 
[44] J. T. Trangenstein, An unsplit Godunov method for three-dimensional polymer flooding, preprint, 1993.

[45] G. Turkiyyah, H. Wu, et al. ZPLCLAW: A portable parallel package for hyperbolic systems of conservation laws. in preparation.

[46] B. van Leer, Towards the ultimate conservative difference scheme IV. A new approach to numerical convection, J. Comput. Phys., 23 (1977), pp. 276-299.

[47] B. van Leer, Towards the ultimate conservative difference scheme $V$. A second order sequel to Godunov's method, J. Compu. Phys., 32 (1979), pp. 101-136.

[48] S. T. Zalesak, Fully multidimensional flux corrected transport algorithms for fluids, J. Comput. Phys., 31 (1979), pp. 335-362.

[49] Viz software. Available on the Web from the URL ftp://ftp.ffi.no/spub/stsk/viz/index.html. 